\title{
Technologies optiques, CD-ROM, et bibliothèques
}

Partie 1: Caractéristiques, marché, applications

Optical Technologies, CD-ROMs and Libraries

Part 1: Characteristics, Markets, Applications

Technologías ópticas, CD-ROM y bibliotecas

Parte I: Características, mercado, aplicaciones

\section{Gilles Deschâtelets et Marcel Simoneau}

Volume 34, numéro 2, avril-juin 1988

URI : https://id.erudit.org/iderudit/1052517ar

DOI : https://doi.org/10.7202/1052517ar

Aller au sommaire du numéro

Éditeur(s)

Association pour l'avancement des sciences et des techniques de la documentation (ASTED)

\section{ISSN}

0315-2340 (imprimé)

2291-8949 (numérique)

\section{Découvrir la revue}

Citer cet article

Deschâtelets, G. \& Simoneau, M. (1988). Technologies optiques, CD-ROM, et bibliothèques : partie 1 : Caractéristiques, marché, applications. Documentation et bibliothèques, 34(2), 43-71. https://doi.org/10.7202/1052517ar
Résumé de l'article

Les auteurs proposent une vue d'ensemble de la nouvelle industrie des technologies optiques et de leurs applications dans le marché de l'information documentaire et notamment, celui des bibliothèques.

Après avoir présenté les aspects techniques et une typologie des nouveaux produits optiques, ils discutent des avantages et des inconvénients du CD-ROM comme support d'information, des opportunités de commercialisation de ce nouveau produit et des caractéristiques du marché des technologies optiques. Ils s'attardent plus particulièrement au marché des bibliothèques et au rôle de ces dernières rôle de consommation et de production face à ce nouveau support.

Dans la seconde partie de cet article, ils présenteront les différents produits disponibles pour les bibliothèques et ils analyseront plus en détail les interfaces et modes d'accès et les méthodes d'évaluation (descriptive, comparative, expérimentale) des produits et systèmes sur le marché.
Tous droits réservés $@$ Association pour l'avancement des sciences et des techniques de la documentation (ASTED), 1988
Ce document est protégé par la loi sur le droit d'auteur. L'utilisation des services d'Érudit (y compris la reproduction) est assujettie à sa politique d'utilisation que vous pouvez consulter en ligne.

https://apropos.erudit.org/fr/usagers/politique-dutilisation/ 


\title{
Technologies optiques, CD-ROM, et bibliothèques
}

\section{Partie 1: Caractéristiques, marché, applications ${ }^{1}$}

\author{
Gilles Deschâtelets* \\ Université de Montréal \\ Marcel Simoneau* \\ Centre canadien de recherche sur \\ l'informatisation du travail
}

Les auteurs proposent une vue d'ensemble de la nouvelle industrie des technologies optiques et de leurs applications dans le marché de l'information documentaire et notamment, celui des bibliothèques.

Après avoir présenté les aspects techniques et une typologie des nouveaux produits optiques, ils discutent des avantages et des inconvénients du CD-ROM comme support d'information, des opportunités de commercialisation de ce nouveau produit et des caractéristiques du marché des technologies optiques. IIs s'attardent plus particulièrement au marché des bibliothèques et au rôle de ces dernières rôle de consommation et de production face à ce nouveau support.

Dans la seconde partie de cet article, ils présenteront les différents produits disponibles pour les bibliothèques et ils analyseront plus en détail les interfaces et modes d'accès et les méthodes d'évaluation (descriptive, comparative, expérimentale) des produits et systèmes sur le marché.

\section{Optical Technologies, CD-ROMs and Libraries}

\section{Part 1: Characteristics, Markets, Applications}

The authors present an overview of the new optical technology industry and the applications in the information marketplace and libraries.

Following a presentation of the technical characteristics and forms of the new optical products and systems, the authors discuss the advantages and limitations of the CD-ROM as an information medium, the marketability of the products and the characteristics of the optic technology market. More specifically, they discuss the library market and the role of the library as consumer and producer of this new product.

In the second part of the article, they will present and discuss various products available to libraries and they will analyse the user interface, the ease of access and the methods of evaluation (descriptive, comparative and experimental) for the products and systems currently available.

\section{Technologías ópticas, CD-ROM y bibliotecas}

\section{Parte l: Características, mercado, aplicaciones}

Los autores proponen una visión general de la nueva industria de las tecnologías ópticas y de sus aplicaciones en el mercado de la información documental, especialmente el de las bibliotecas.

Después de presentar los aspectos técnicos y una tipología de los nuevos productos ópticos, discuten las ventajas y los inconvenientes del CDROM como soporte de datos, las oportunidades de comercialización de este nuevo producto y las características del mercado de las tecnologías ópticas. Tratan particularmente del mercado y del papel de consumo $y$ de producción de las bibliotecas frente a este nuevo soporte de datos.

En la segunda parte de este artículo, los autores presentarán los distintos productos disponibles para las bibliotecas y analizarán en detalle las interfaz, los modos de acceso, los métodos de evaluación (descriptiva, comparativa, experimental) de los productos y de los sistemas disponibles en el mercado.

\footnotetext{
* Gilles Deschâtelets est professeur agrégé à l'École de bıbliothéconomı et des sciences de l'informatıon de l'Unıversité de Montréal, Marcel Sımoneau est chargé de recherche au Centre canadien de recherche sur linformatisation du travail du Ministère des Communications du Canada.
}

1. Dans cette partie, nous nous sommes inspérés d'un document de Jake $\mathrm{V}$ TH. Knoppers et Marcel Simoneau, Le CD-ROM et autres disques compacts: caractéristiques, production et marché, Laval, CCRIT, 25 août 2987, 35p. 
Les nouvelles technologies optiques sont en voie de révolutionner l'industrie de l'information nordaméricaine et même européenne, en remettant en question certains produits et services utilisés jusqu'à maintenant comme supports privilégiés d'information. Ainsi, les nouvelles technologies optiques entrent directement en compétition avec certains produits documentaires imprimés (ouvrages de référence, répertoires signalétiques et analytiques, périodiques, etc.) et avec certains services de stockage et de diffusion de l'information (service de téléréférence, services d'archivage ordinolingue, catalogues ordinolingues de bibliothèques, etc.). À cette information textuelle, les nouvelles technologies optiques permettent et permettront de plus en plus d'intégrer le stockage et la diffusion d'information non textuelle, comme le son et l'image (fixe et animée).

Si la confrontation n'a pas encore vraiment eu lieu, c'est que le marché est toujours en gestation, autant en ce qui touche les matériels (équipements, logiciels, interfaces) que les produits et les clientèles visées. Mais la collision semble inévitable et les bibliothèques, qui sont encore au coeur des activités d'information documentaire, en seront des acteurs privilégiés. II importe donc, afin de maximiser l'implantation de ce nouveau support d'information dans les bibliothèques, que celles-ci soient bien au fait de ses caractéristiques, de ses avantages et de ses limitations, et surtout, des produits et des applications adaptés et adaptables à ce milieu.

Le présent article vise donc d'abord à présenter aux spécialistes de l'information documentaire, cette nouvelle technologie, son marché, ses produits et les applications plus particulièrement intéressantes pour les bibliothèques. Dans un second article, nous analyserons plus attentivement les modes d'accès aux bases de données sur CD-ROM en les comparant notamment à la recherche documentaire manuelle et à la téléréférence.

\section{Disques optiques: aspects techniques \\ Origines du CD-ROM}

La technologie à l'origine du CD-ROM (compact disc-read only memory) provient surtout de l'industrie des équipements électroniques de loisir, dû aux nombreuses caractéristiques techniques empruntées au disque compact audio. Cependant, les CD-ROM se distinguent par l'apport de l'industrie de l'informatique pour la mise au point de logiciels nécessaires à l'accès et à la recherche interactive des données mémorisées. On peut brièvement en retracer l'évolution:

\section{9}

Philips entreprend des recherches pour l'enregistrement d'images, de son et de données textuelles sur disque optique en utilisant le haut degré de précision du rayon laser.

\section{5}

Philips manufacture les premiers prototypes de vidéodisques pouvant contenir des images par un procédé de lecture au laser.

\section{8}

Lancement du premier vidéodisque sous le nom du système Laser Vision. Le disque compact audio est défini dans sa forme finale, soit une heure de musique sur un disque de $12 \mathrm{~cm}$ de diamètre. Entente entre Philips et Sony sur l'établissement d'une norme industrielle mondiale pour les disques compacts.

\section{0}

Le concept du CD-ROM prend de l'ampleur lorsque la faisabilité et l'acceptation du CD-audio se concrétisent.

\section{1}

Un disque compact audio est présenté comme prototype au festival international du son à Paris.

\section{3}

Présentation d'un premier prototype de disque effaçable selon le procédé du changement de phase, par la firme Matsushita.

\section{$1983 / 1984$}

Mise en marché, par Philipps et Sony, des premiers disques compacts et des lecteurs au laser.

\section{4}

Philips présente les prototypes CD-ROM.

\section{5}

Mise en marché des premières bases de données sur CD-ROM et de leurs appareils de lecture. Verbatim (une filiale de Kodak) présente publiquement un disque magnéto-optique (DMO) d'une capacité de 50 méga-octets pour un format de 3,5 pouces

\section{6}

Première annonce, par Philips et Sony, d'un CDI (compact disc interactive) à la 1 ère conférence annuelle de Microsoft. Mise sur pied, par les industries concernées, d'un groupe de travail, le High Sierra Group (HSG), pour l'établissement de normes minimales pour la production de disques CD-ROM.

\section{$1986 / 1987$}

Participation accrue des éditeurs et distributeurs dans la commercialisation de produits optiques (bases de données et ouvrages de référence sur CD-ROM) à grande échelle. 


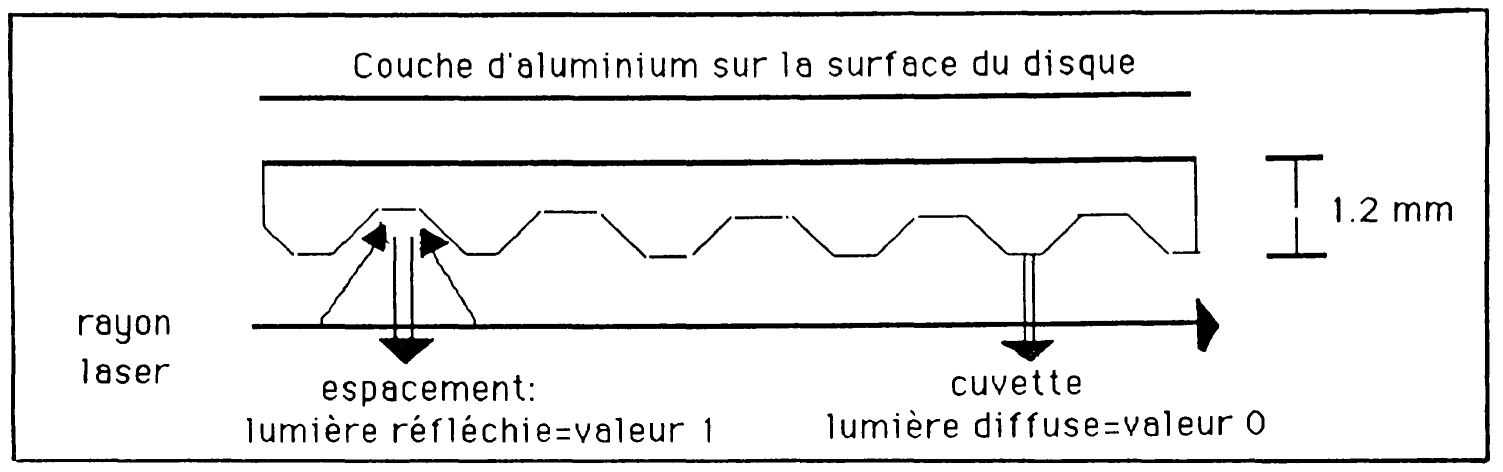

Fig. 1 : CD-ROM : conversion des signaux lumineux en code binaire

\section{7}

Présentation, par la firme Digital Audio Disc Corp (une filiale de Sony) d'un prototype CD-V (compact disc)video) contenant 5 minutes de vidéo et 20 minutes de musique. La firme $3 \mathrm{M}$ présente un DMO de 5,25 pouces avec une capacité de 200 à 400 méga-octets par face. La firme Maxtor de Californie présente des DMO prototypes de 5,25 pouces avec 760 méga-octets et de 3,5 pouces avec 170 méga-octets. Première démonstration, par General Electric, d'un digital video-interactive (DV-1), à la 2ième conférence annuelle de Microsoft. Le 11 daéacembre, première démonstration en Amérique du Nord d'un CD-I par Philips.

\section{8}

Acceptation des normes HSG par la National Information Standards Organisation (NISO), par I'European Computer Manufacturers Association (ECMA) et par I'International Organization for Standardization (ISO). La norme est acceptée par la plupart des manufacturiers. Au printemps, Tandy Corporation annonce le Tandy THOR-CD, un disque compact sur lequel les données audio ou textuelles ou vidéo peuvent être enregistrées et effacées par l'utilisateur. Présentation, au printemps, par Discovery System, d'un CD-ROM prototype qui intègre images, texte et son et qui peut être lu sur un lecteur de CD-ROM. Mise en marché prévue pour l'automne des premiers $C D$ $\mathrm{I}$, des premiers DMO et de deux modèles de lecteur, par la firme Maxtor. Production prévue pour la fin de l'année, par la firme Olympus, d'une unité de disque effaçable à changement de phase, d'un format de 5,25 pouces et de 240 méga-octets de capacité.

\section{9}

Mise en marché prévue des premiers «compact disc-write once» (CD-WO), format de 4,72 pouces, compatibles avec les CD-ROMs. Mise en marché prévue, par la firme Creo Products Inc. de Vancouver, des premiers lecteurs de «digital paper» (DP). Mise en marché prévue, par la firme Nikon, d'une unité de lecture de DMO.

\section{Mode de fonctionnement d'un CD-ROM}

\section{Enregistrement des données}

Un CD-ROM est un disque de polycarbonate de $12 \mathrm{~cm}$ de diamètre, de $1,2 \mathrm{~mm}$ d'épaisseur, avec un trou au centre de $15 \mathrm{~mm}$. L'information est enregistrée sur une spirale ou un cercle concentrique de sillons formés par l'alternance de trous et d'espaces. Les trous, appelés cuvettes ou microcuvettes, sont formés par la brûlure d'un rayon laser sur la surface vierge du disque. Les cuvettes ont 12 microns de profondeur et 6 microns de largeur et sont séparées par des espaces de 1,6 micron.

Ces espacements correspondent à une densité de 600 sillons par $\mathrm{mm}$. C'est la forte densité de ces sillons qui permet au CD-ROM de contenir un volume énorme d'espace mémoire pour les données codées. En comparaison, une disquette contient 4 sillons par mm et un disque dur, environ 100 sillons par $\mathrm{mm}$.

\section{Lecture des données}

Les CD-ROMs sont lus par un rayon laser de faible intensité ( 2 à 3 milliwatts) d'où l'appellation "disque au laser». Le faisceau du rayon balaie les sillons du disque. Lorsque la lumière éclaire les cuvettes, elle se disperse dans tous les sens. Mais lorsqu'elle vise un espacement, celui-ci réfléchit le rayon directement sur une lentille orientée vers un photodétecteur, produisant ainsi un signal lumineux. C'est le signal modulaire produit par la combinaison de lumière diffuse (valeur " $0 "$ ) et de lumière réfléchie (valeur "1») qui représente l'information enregistrée sur le disque. Cette information produite par la modulation d'un signal lumineux est convertie en données numériques (forme binaire) pouvant être reconnues par l'ordinateur. 


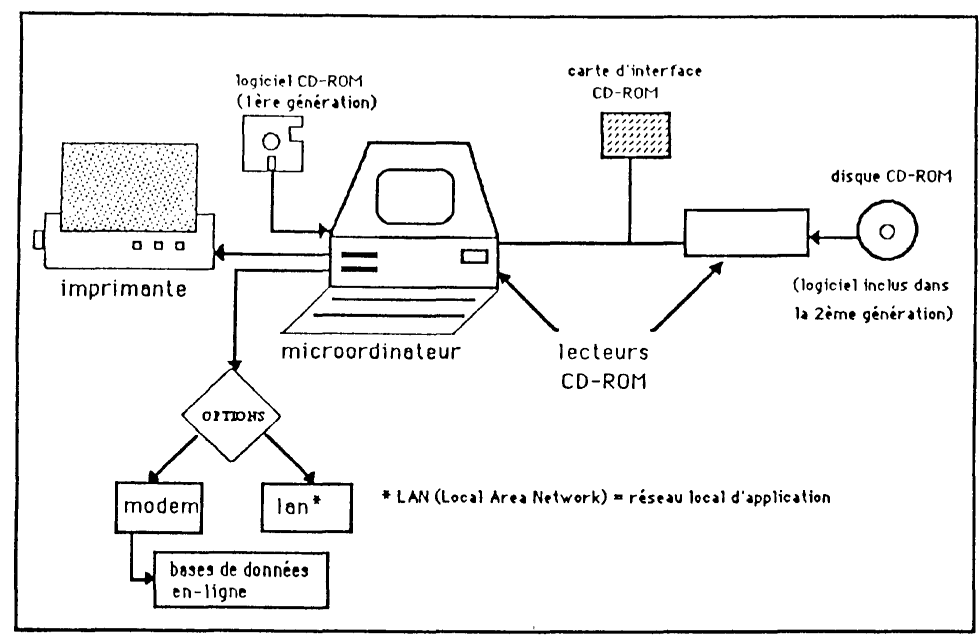

Fig. 2 : Poste de trovoil pour CD-ROM

\section{Transmission des données}

L'exploitation des données stockées sur CD-ROM se fait au moyen d'un lecteur optique relié à un microordinateur. La figure 2 décrit la configuration d'un poste-type de travail pour CD-ROM.

Les données sont lues par un rayon laser dans le lecteur optique, elles sont converties sous forme binaire grâce à la carte d'interface dans le microordinateur et elles sont ensuite "appelées» par le logiciel de recherche, d'où le lien essentiel entre le CD-ROM et le microordinateur. L'accès à une donnée précise est possible par la division du volume total d'information en un certain nombre de fichiers interrogeables chacun au moyen d'index, un peu comme sur un disque dur. Le logiciel de recherche permet de choisir un mot-clé dans l'index et de trouver les enregistrements selon une hiérarchie correspondant au mot-clé. Si le mot-clé choisi par l'utilisateur n'est pas dans l'index, le logiciel trouvera le mot le plus près selon un ordre alphabétique «lettre par lettre» ou permettra à l'utilisateur de faire un «balayage» dans l'index afin de trouver un synonyme.

La qualité de l'indexation influence directement la performance du logiciel à retrouver, de façon rapide et précise, les données enregistrées sur le CD-ROM. Les index occupent en général de 35 à $50 \%$ du volume total d'espace sur un CDROM; dans certains cas, cela peut même aller jusqu'à $75 \%$ de l'espace. En fait, la taille de l'index augmente proportionnellement avec celle de la base de données.

En somme, à l'instar des autres supports d'information électronique (magnétiques), le logiciel d'interrogation joue un rôle essentiel d'intermédiaire entre l'utilisateur et le disque CDROM.

\section{Disques optiques: typologie des produits}

Un bref rappel des différents produits optiques déjà commercialisés ou annoncés et une présentation sommaire de leurs caractéristiques permettra au lecteur de mieux s'y retrouver dans cette nouvelle jungle technologique et terminologique ${ }^{2}$.

\section{Avantages du CD-ROM et des supports optiques}

Le CD-ROM et les autres supports optiques offrent aux bibliothèques et aux centres de documentation des avantages importants: 1) leur grande capacité de stockage comparée, notamment, aux supports imprimés et magnétiques; 2 ) la diminution du coût de stockage de l'information; 3) le principe de tarification sous forme d'abonnements annuels fixes qui facilite la budgétisation; 4) la durabilité, la permanence de l'information et son insensibilité aux champs magnétiques; 5) l'autonomie locale de recherche accrue; 6) le stockage de textes complets; 7) le mariage du texte, du son et de l'image; 8 ) les coûts initiaux relativement peu élevés; 9) la facilité d'utilisation des équipements et des bases de données; 10) la facilité de manipulation et de transport des équipements et des bases de données; 11) l'économie d'espace; 12) les effets d'entraînement sur le service de téléréférence et sur les recherches en-ligne; 13) l'exploitation du CDROM améliore l'image de la bibliothèque et des bibliothécaires et attire de nouvelles clientèles. Voyons ces avantages plus en détail.

CAPACITE DE STOCKAGE. Nous n'insisterons pas sur la voracité de l'imprimé à consommer les espaces des bibliothèques. Quant aux supports magnétiques, ils ne font tout simplement pas le poids, en terme de stockage, face aux supports optiques, comme on peut le voir à la figure 5 . 
$\dot{A}$ titre d'exemple, en termes de comparaison, un disque optique CD-ROM a la même capacité de stockage que 297000 pages $81 / 2^{\prime \prime}$ x 11 " dactylographiées à double interligne, ou 1500 disquettes magnétiques de 5 ,» de 360 kilo-octets, ou encore 55 disques durs magnétiques de 10 méga-octets chacun. On calcule, par ailleurs, qu'un seul disque CD-ROM peut contenir l'équivalent de 46 jours complets de recherche en ligne à 1200 bauds ou du texte d'une secrétaire professionnelle (90 mots à la minute) tapé sans arrêt à raison de 8 heures par jour, 5 jours par semaine, pendant 8 ans et 3 mois... soit 70 millions de mots! Cette énorme capacité de stockage fait que l'accès à l'information est beaucoup plus rapide qu'avec une disquette ou un microfilm, tout simplement parce qu'il y a beaucoup moins de supports à manipuler pour chaque recherche.

COÛT DE STOCKAGE. Le coût de stockage de l'information sur supports optiques a diminué de facteur 5 depuis 1983. A moyen terme, cela devrait avoir des répercussions heureuses sur la prolifération de nouveaux produits et sur leurs coûts.

Le coût de revient de stockage de l'information sur CD-ROM est le plus bas de tous les supports d'information à l'heure actuelle: \$0,02 par million de caractères, soit environ $1 / 500$ des coûts de stockage sur papier ou sur disquette. II faut toutefois, pour atteindre la rentabilité, un énorme volume d'information à stocker ( 500 millions de caractères ou plus) et une distribution assez élevée (de l'ordre de 1000 copies ou plus) 3 .

TARIFICATION. Le principe de tarification de ces nouveaux produits, notamment des bases de données commerciales sur CD-ROM, sous forme d'abonnements annuels fixes, facilite la budgétisation pour les bibliothèques. L'on se rapproche ici davantage du mode d'abonnement aux répertoires imprimés que de celui aux services d'interrogation en ligne basé sur le volume d'utilisation, souvent difficile à planifier.

DURABILITÉ DE L'INFORMATION. La durabilité, la permanence de l'information enregistrée sur les supports optiques et son insensibilité aux champs magnétiques constituent un avantage certain. Pour la majorité des produits disponibles, les données ne peuvent être ni effacées, ni même inscrites par l'utilisateur. De par leur structure physique, les supports optiques ont une espérance de vie de près du double (10-20 ans) de celle des supports magnétiques (5-10 ans). En outre, ce sont des produits relativement robustes qui se prêtent bien à une manipulation intensive.

AUTONOMIE DE RECHERCHE. L'autonomie locale de recherche est accrue dû au fait que la tarification des recherches bibliographiques, contrairement aux services d'interrogation en ligne, n'est plus fonction du temps de connexion. L'usager, quel qu'il soit, ne subit donc plus la pression du tarif horaire et peut donc effectuer ses recherches beaucoup plus librement et calmement.

STOCKAGE DE TEXTES COMPLETS. Cette caractéristique inverse, en quelque sorte, la démarche traditionnelle de la recherche documentaire. Même si elle n'est pas exclusive aux technologies optiques (on trouve des textes complets sur supports magnétiques), elle n'en demeure pas moins fort importante pour la recherche documentaire 4 . Selon la démarche "classique», on repère d'abord des références bibliographiques, puis les documents "référés» et enfin, l'information ou les unités d'information désirées. L'accès direct au texte permet (et permettra de plus en plus) d'inverser ce processus et de repérer immédiatement les unités d'information désirées sans vraiment passer par les deux premières étapes. Ce sera, si le processus se généralise, la véritable révolution de la science de l'information et l'apport des technologies optiques à cette mutation sera capital.

MARIAGE DU TEXTE, DU SON ET DE L'IMAGE (FIXE ET ANIMÉE). Ce mariage permettra aux supports optiques d'atteindre leur véritable potentiel. Jusqu'à présent, en effet, les technologies optiques ont surtout été exploitées en documentation comme supports de masse pour des applications textuelles. Ce transfert technologique des supports magnétiques aux supports optiques, s'il favorise la création d'une nouvelle industrie en mettant en marché des produits déjà connus auprès de clientèles-cibles également identifiées, n'en restreint pas moins son immense potentiel à une seule dimension, le texte. L'originalité et l'avenir des technologies optiques résident plutôt dans leur capacité à marier harmonieusement le texte, le son et l'image, non seulement au stockage (encyclopédies audioscripto-visuelles) mais également au repérage (langages iconiques, synthèse et reconnaissance de la parole permettant une véritable «conversation» avec la machine et, partant, une «entrevue de référence» entre la machine et l'utilisateur $)^{5}$

3. Peter Schipma et Lois F. Lunin, «Perspectives on...CD-ROM for information retrieval. Introduction and overview», Journal of the American Society for Information Science. vol. 39, no. 1 (January 1988), 31-33

4. Voir aussi Gilles Deschâtelets, «De la bibliothéconomie à la science de l'information ou l'information libérée dy carcan documentaire", Argus, vol. 16, no 1 (mars 1987), 21-26.

5. Cette chronologie existe d'ailleurs déjà avec le Fiction Advisor du Groupe Del Mar, qui conseille I'utilisateur sur le choix d'un roman, et avec le Intelligent Catalog de la Library Corporation. Voir à ce sujet l'article de Richard A Bowers, "CD-ROM computer theater: the play's the thing", CD-ROM Review, vol. 3, no. 5 (July 1988), 10-11. En outre, la compagnie Apple vient d'annoncer son projet du Whole Earth Catalog sur CD-ROM avec son Maclntosh et le logiciel Hypercard (MacUser, (June 1988), 157-167. 
Figure 3: Disques optiques - Typologie des produits

\section{Caractéristiques}

\begin{tabular}{|c|c|c|c|c|c|}
\hline \multirow{2}{*}{$\begin{array}{l}\text { Produits/ } \\
\text { Technologies }\end{array}$} & & & & & \multirow[b]{2}{*}{ Exemple } \\
\hline & $\begin{array}{l}\text { Type de } \\
\text { document }\end{array}$ & Mode de codage & Capacité & $\begin{array}{l}\text { Pérennité des } \\
\text { informations }\end{array}$ & \\
\hline VIDEODISQUE & $\begin{array}{l}\text { Images fixes } \\
\text { Images animées } \\
\text { Son } \\
\text { Texte } \\
\text { Donnée } \\
\text { numériques }\end{array}$ & $\begin{array}{l}\text { Analogique } \\
\text { Numérique }\end{array}$ & $200 \mathrm{mo}$ & Ineffaçable & $\begin{array}{l}\text { DiskPlay de } \\
\text { Techniform } \\
\text { International }\end{array}$ \\
\hline CD-ROM & $\begin{array}{l}\text { Texte } \\
\text { Données } \\
\text { numériques }\end{array}$ & Numérique & $540 \mathrm{mo}$ & Ineffaçable & Silver Platter \\
\hline CD-I & $\begin{array}{l}\text { Images fixes } \\
\text { Son } \\
\text { Texte } \\
\text { Données } \\
\text { numériques }\end{array}$ & Numérique & & Ineffaçable & $\begin{array}{l}\text { Prototype de } \\
\text { Philips }\end{array}$ \\
\hline CD-WORM & $\begin{array}{l}\text { Images fixes } \\
\text { Texte } \\
\text { Données } \\
\text { numériques }\end{array}$ & Numérique & $200 \mathrm{mo}$ & Ineffaçable & I B M \\
\hline CD-V & $\begin{array}{l}\text { Images fixes } \\
\text { Images animées } \\
\text { Son } \\
\text { Texte } \\
\text { Données } \\
\text { numériques }\end{array}$ & $\begin{array}{l}\text { Analogique } \\
\text { Numérique }\end{array}$ & & Ineffaçable & $\begin{array}{l}\text { Prototype de } \\
\text { Sony (1987) }\end{array}$ \\
\hline DVI & $\begin{array}{l}\text { Images fixes } \\
\text { Images animées } \\
\text { Son } \\
\text { Texte } \\
\text { Données } \\
\text { numériques }\end{array}$ & $\begin{array}{l}\text { Analogique } \\
\text { Numérique }\end{array}$ & & Ineffaçable & $\begin{array}{l}\text { Prototype de } \\
\text { General } \\
\text { Electric }\end{array}$ \\
\hline DMO & $\begin{array}{l}\text { Images fixes } \\
\text { Texte } \\
\text { Données } \\
\text { numériques }\end{array}$ & Numérique & $\begin{array}{l}\text { de } 100 \mathrm{mo} \\
\text { à } 1 \mathrm{go}\end{array}$ & $\begin{array}{l}\text { Effaçable } \\
\text { réinscriptible }\end{array}$ & $\begin{array}{l}\text { Maxtor } \\
\text { (automne 88) }\end{array}$ \\
\hline CARTE LASER & $\begin{array}{l}\text { Texte } \\
\text { Données } \\
\text { numériques }\end{array}$ & Numérique & $16 \mathrm{mo}$ & Ineffaçable & $\begin{array}{l}\text { Drexon } \\
\text { Lasercard de } \\
\text { Drexler }\end{array}$ \\
\hline
\end{tabular}


Figure 4: Glossaire des principales technologies optiques

CD (compact disc): disque optique d'un format standard de 4,72 pouces ou $12 \mathrm{~cm}$, utilisé comme support de données audio ou textuelles numérisées.

CD-DA (compact disc digital audio): norme industrielle $(4,72 \mathrm{po})$ à laquelle sont conformes tous les disques compacts audio et tous les lecteurs de disques compacts audio. Connue aussi sous le nom de RED BOOK standard.

CD-I (compact disc-interactive): nouveau disque compact $(4,72$ po), prévu sur le marché pour la fin de 1988 et qui offrira des capacités intégrées de lecture de données audio, infographiques et textuelles. Un lecteur de disque $C D-I$ pourra lire aussi bien un disque compact audio, CD-ROM et CD-I.

CD-ROM (compact disc-read only memory): norme de format de disques compacts 14,72 po) qui permet un volume de stockage d'environ 550 méga-octets de données. Les données sur les CD-ROMs peuvent être lues seulement, sans possibilité d'effaçage ou de réinscription.

CD-V (compact disc-video): norme de format de disque compact $(4,72$ po) qui permet le stockage de 5 minutes de video (codage analogique) et environ 20 minutes de son stéréo de qualité (codage numérique).

CD-WO (compact disc-write once): nouvelle version de CD-WORM de 4,72 pouces qui permettra l'enregistrement de données sur un disque compact vierge, sans possibilité d'effacement. Les systèmes CD-WO se distingueront des CD-WORMs actuels en ce qu'ils pourront aussi lire des CD-ROMs.

CD-WORM (compact disc-write once read many): disque compact sur lequel les données, dès qu'elles sont inscrites par l'usager, ne peuvent plus être effacées. Les CD-WORMs sont disponibles en formats de 14 pouces, 12 pouces, 5,25 pouces et 3,5 pouces. Le volume de stockage peut varier de plusieurs mégaoctets à plus de 4 giga-octets. Les lecteurs de disques ou de cassettes CD-WORMs ne peuvent pas lire des disques CD-ROMs.
DMO (disque magnéto-optique): disque optique effaçable, encore à l'état de prototype, tirant son nom de la technologie utilisée pour effacer et réinscrire les données. La plupart des fabricants semblent vouloir opter pour ce procédé.

DOE (disque optique effaçable): disque optique au format de 3,5 pouces et 5,25 pouces, encore à l'état de prototype. Les données sur ces disques pourront être effacées et réinscrites plusieurs fois par l'utilisateur. Pour ce faire, deux technologies différentes sont utilisées: magnéto-optique et changement de phase.

DV-I (digital video-interactive): nouvelle technologie introduite par General Electric et RCA qui permettra à un lecteur CD-ROM de lire des données audio et vidéo sur un disque compact de 4,72 pouces. En ce sens, cette technologie est semblable à celle du CD-I, mais elle permettra une plus grande durée de lecture vidéo grâce à des meilleurs taux de compression des données (de 7 à 21).

D P (digital paper): nouvelle technologie en développement qui permettra de stocker sur un mince ruban de polyuréthane des données numériques. Conçu spécifiquement pour des fins d'archivage, un système de stockage utilisant ce type de ruban pourra contenir jusqu'à 1 tétra-octet de données, soit l'équivalent de 1600 disques CD-ROMs. Les données enregistrées ne pourront toutefois pas être effacées.

Green Book: nom donné au standard du CD-I.

HGS (High Sierra Standard): format standard qui spécifie l'organisation des fichiers et des répertoires sur les disques CD-ROMs. Ce format a été défini par un groupe d'industries de l'informatique et des technologies optiques.

IVD (interactive video disc): connue aussi sous le nom de LaserVision, cette technologie permet le visionnement de films sonores en mode interactif, sur un disque optique de 12 pouces. Le vidéodisque est surtout utilisé à des fins d'éducation et de formation.

Jukebox: système de stockage dans lequel plusieurs disques WORMs peuvent être empilés, permettant ainsi des capacités d'archivage de données de plusieurs giga-octets. 
Figure 4 (suite)

Lasercard (carte au laser): carte de polyuréthane sur laquelle jusqu'à 16 méga-octets de données numériques textuelles peuvent être emmagasinées sans toutefois pouvoir être effacées.

MS-DOS Extension: conçu par Microsoft, ce programme informatique permet à tout lecteur de CD-ROM répondant aux standards HGS d'être "reconnu» par le DOS d'un microordinateur. Le lecteur CD-ROM est alors considéré comme un disque dur.OCR (optical character recognition): technologie qui permet à un balayeur optique (scanner) de numériser de l'information à partir de supports imprimés pour la transférer dans un ordinateur.

OCR (optical character recognition): technologie qui permet à un balayeur optique (scanner) de numériser de l'information à partir de supports imprimés pour la transférer dans un ordinateur.
OROM (optical read only memory): disque compact d'un format de 5,25 pouces, utilisé surtout par les forces militaires. Ce format n'est pas normalisé.

Red BOOK: nom donné au standard du disque compact audio (CD-DA).

SCSI (small computer system interface) interface qui permet de connecter en mode parallèle des périphériques tels des lecteurs optiques à des microordinateurs. Tout lecteur équipé d'une interface SCSI peut être connecté à un microordinateur (IBM ou Apple) qui comprend une interface compatible.

Vidéodisque: voir IVD.

Yellow Book: nom donné au standard du CDROM, tel que défini par Sony et Philips. Contient les spécifications pour le stockage de données numériques sur CD-ROM.

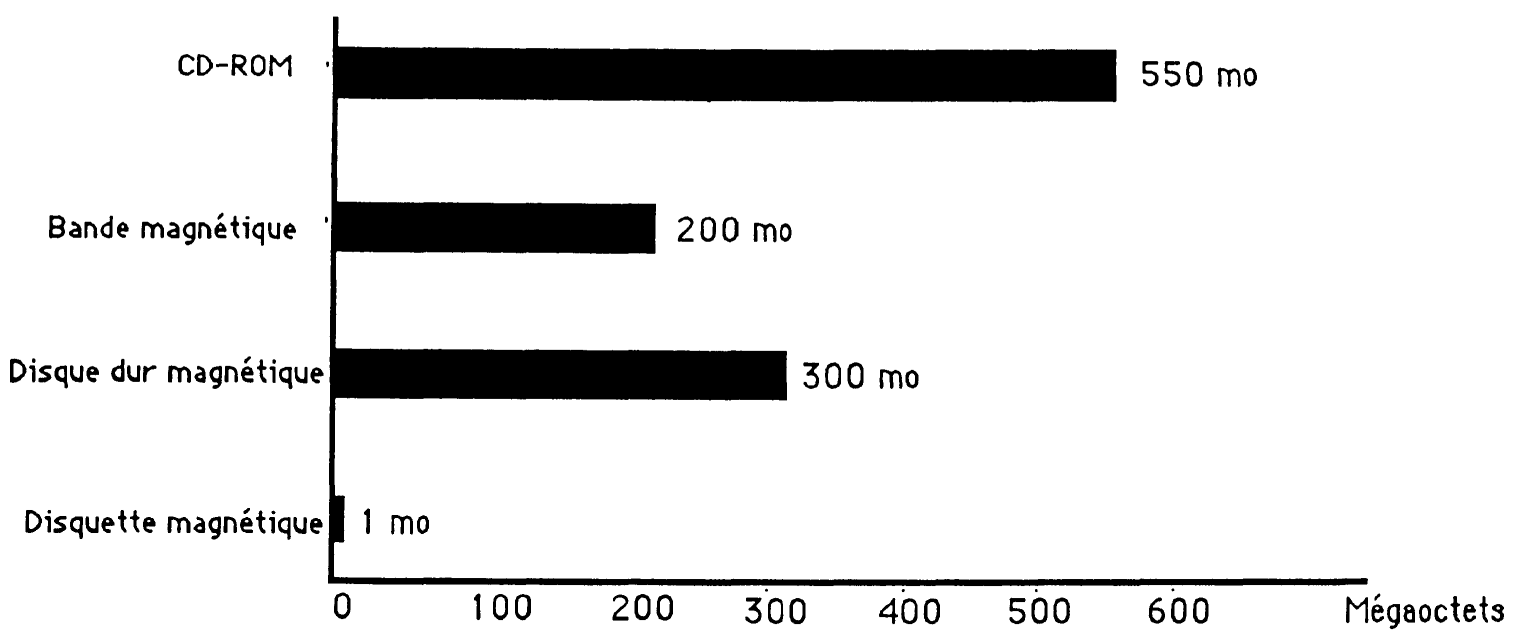

Fig.5: Capacité moyenne de stockage de divers supports d'information 
COÛTS INITIAUX. Ces coûts sont relativement peu élevés. Les équipements requis sont: un microordinateur, un lecteur optique et une carte d'interface. À l'exclusion du micro-ordinateur, le coût d'achat du lecteur optique et de la carte d'interface devrait se situer entre $1000 \$$ et 1 $500 \$$. Ces coûts devraient d'ailleurs diminuer légèrement dans les prochaines années ( $1000 \$$ ).

FACILITÉ D'UTILISATION. Les équipements sont, en effet, très simples à utiliser, une fois que la carte d'interface est installée (aussi simples que les lecteurs de disques compacts audio pour la plupart). Quant aux bases de données, leur facilité d'exploitation et leur convivialité varie considérablement d'un produit à l'autre. II faut toutefois distinguer les bases de données des ouvrages de référence et autres produits lorsqu'on parle des modes d'accès. Ceux-ci diffèrent d'une catégorie à l'autre ${ }^{6}$. En général, les bases de données sur CD-ROM sont presque toutes accessibles en mode «menu» et ne requièrent donc que très peu d'apprentissage au niveau des langages d'interrogation. II n'en va pas toujours de même, cependant, avec le langage d'indexation et la structure de la base de données.

FACILITÉ DE MANIPULATION ET DE TRANSPORT. Cette maniabilité des bases de données est souvent indispensable à cause des contraintes d'utilisation des équipements qui sont encore largement mono-postes et mono-utilisateurs.

ÉCONOMIE D'ESPACE. L'économie d'espace réalisée est considérable par rapport aux ressources imprimées correspondantes (notamment les répertoires analytiques).

EFFETS D'ENTRAÎNEMENT. Si plusieurs auteurs notent une diminution du volume des recherches en ligne, principalement les recherches courtes et élémentaires, suite à l'exploitation de bases de données sur CD-ROM, plusieurs soulignent également une augmentation des recherches plus complexes et médiatisées. Les bases de données sur CD-ROM sont également utilisées pour vérifier et ajuster des stratégies de recherche avant d'aller en ligne.

VISIBILITÉ DE LA BIBLIOTHĖQUE. Finalement, à I'instar du service de téléréférence à ses origines, l'exploitation des bases de données sur CD-ROM donne à la bibliothèque une visibilité et un certain respect de sa clientèle. Ce nouveau service améliore l'image de la bibliothèque et des biblio- thécaires. En outre, les expériences rapportées dans les écrits soulignent que ce service attire de nouvelles clientèles (notamment les étudiants de premier cycle dans les universités).

\section{Inconvénients du CD-ROM et des supports optiques}

Le CD-ROM possède toutefois quelques faiblesses et certains inconvénients parfois ennuyeux. Reese et Steffey parlent des «sept péchés capitaux du CD-ROM»? Voici les principaux inconvénients.

TECHNOLOGIE DE STOCKAGE ET D'ACCĖS BEAUCOUP PLUS LENTE que celle des disques magnétiques. Par exemple, les temps de recherche sur CD-ROM sont de 5 à 20 fois plus lents que ceux d'un disque dur magnétique de type Winchester. Par ailleurs, comme les délais de télécommunications sont éliminés, le problème s'en trouve quelque peu amoindri par rapport à la recherche en ligne. D'autre part, les nouveaux lecteurs de 2ième génération sont déjà beaucoup plus rapides que les premiers.

INSTALLATION PARFOIS DIFFICILE ET DÉLICATE, surtout avec un disque dur et plusieurs bases de données utilisant des logiciels d'interrogation différents. II existe encore de nombreux problèmes de compatibilité.

MARCHÉ ET TECHNOLOGIE EN MUTATION ACCÉLÉRÉE. Certains produits apparaissent et disparaissent tout à coup. La quincaillerie évolue à un rythme effréné. Certains produits apparaissent en plusieurs versions différentes (ex: il existe 6 différentes versions MEDLINE sur CD-ROM). Les normes ont longtemps été inexistantes ou en phase d'élaboration, aussi bien pour le matériel (lecteurs, disques) que pour les interfaces, les logiciels et les contenus. Cette question des normes d'équipements et de produits est toutefois bien proche d'être résolue. En effet, les manufacturiers de lecteurs optiques semblent vouloir adopter la norme d'interface SCSI qui fait que I'on pourra connecter toute marque de lecteur avec son microordinateur. Par ailleurs, les producteurs d'information sur CD-ROM ont adopté la norme HGS pour l'organisation physique des fichiers et des répertoires. Jusqu'à présent, certains disques n'étaient pas compatibles avec certains lecteurs ${ }^{8}$. Enfin, chaque producteur de disque utilise un logiciel de repérage et un langage d'interrogation différents.
6. Nous verrons plus en détail les différents modes d'accès dans la seconde partie de l'article.
7. Jean Reese et Ramona J Steffey, «The seven deadly sins of CD-ROM", Laserdisk Professional, vol. 1, no. 2 (July 1988), 19-24.

8. Voir à ce sujet l'article de Mike Befeler et John Einberger, "CD-ROM standardization», Laserdisk Professional, vol. 1, no. 2 (July 1988), 70-72. 
RECHERCHES MULTICRITĖRES GÉNÉRALEMENT LIMITEEES. La plupart du temps, les logiciels de repérage ne permettent que 2 ou 3 critères (conditions de repérage: «et" ou «ou» logiques) par recherche. Les recherches doivent être très simples ou alors morcelées en de nombreuses expressions. Souvent, la puissance du microordinateur et du langage d'interrogation limitent sérieusement l'étendue des recherches possibles.

LECTEUR PRESQUE EXCLUSIVEMENT MONOPOSTES ET MONO-UTILISATEURS, c'est-à-dire qu'un seul usager peut consulter une seule base de données sur un seul lecteur à la fois.

RECHERCHES EN MODE CONVIVIAL (MENU, GRAPHIQUE) GÉNÉRALEMENT PLUS LONGUES QU'EN MODE EXPERT (COMMANDES). II y a davantage d'étapes pour une même stratégie (jusqu'à 4 pour 1 dans certains cas) en mode convivial, la s:́ratégie devant être très explicite.

MULTIPLICITÉ DES FICHIERS POUR UNE MÊME BASE DE DONNÉES. Alors que la tendance, dans la recherche en ligne sur grands serveurs est justement de consolider les bases de données, celles sur CD-ROM, à cause de leur capacité de stockage (l'index peut occuper jusqu'à $75 \%$ du disque) et pour des raisons évidentes de profit, sont plutôt morcelées (par année, par section) et constituent des produits distincts, vendus séparément.

UTILISATEUR DÉPENDANT DU PRODUCTEUR DE BASES DE DONNÉES. C'est, en quelque sorte, un transfert de dépendance du serveur au producteur de bases de données. Cela entraîne un certain danger d'anarchie dans les langages d'interrogation, s'il n'y a pas de concertation et d'efforts des producteurs pour les normaliser. Le problème est que l'on vise à la fois et avec les mêmes produits, les utilisateurs novices et les experts professionnels.

FRÉQUENCE DES MISES À JOUR BEAUCOUP PLUS LENTE QUE POUR LA RECHERCHE EN LIGNE. La tendance actuelle est une mise à jour trimestrielle pour les bases de données sur CDROM alors qu'elle est plutôt mensuelle pour les produits en ligne. Souvent, il existe des options de mises à jour, mais plus la fréquence augmente, plus le coût de l'abonnement augmente également.

COÛTS DES ABONNEMENTS. Les coûts d'abonnements annuels peuvent être très élevés (dépendant du type de produit et de la fréquence des mises à jour). II s'agit de coûts fixes quine tiennent pas compte du degré d'utilisation.

COÛT ENCORE TRĖS ÉLEVÉ DE PRODUCTION D'UN DISQUE MAITRE. Pour qui voudrait produire un fichier sur CD-ROM, il faudrait compter entre 4000 s et 25 O00\$. PEU D'ILLUSTRATIONS, D'IMAGES ET DE GRAPHIQUES dans les pre- mières bases de données sur CD-ROM. En 1987, toutefois, on commence à voir apparaître des produits «illustrés»: Biolite de Microtrends, le World Atlas de Delorme Mapping System, Geovision de Norcross et en 1988, le Discovery System.

NOMBRE ENCORE RELATIVEMENT RESTREINT DE BASES DE DONNÉES. II existe à peine 300 bases de données disponibles sur CD-ROM comparativement aux 3600 disponibles en ligne.

NOMBREUX FORMATS NON COMPATIBLES. LeS différents formats (CD-ROM, CD-I, CD-WORM) exigent non seulement des supports mais même des équipements différents. Beaucoup de recherches sont en cours, cependant, pour régler ces questions de compatibilité. Ainsi, les lecteurs CD-ROM de 2 ième génération peuvent lire aussi bien les disques CD-ROM que les disques CDaudio. Par ailleurs, on annonce pour bientôt une compatibilité entre les CD-WORM et les CD-ROM grâce à la nouvelle technologie du CD-WO.

\section{Opportunités commerciales du CD-ROM}

Les disques optiques - et notamment le CD-ROM constituent une alternative intéressante aux supports «traditionnels" de l'information, tant électroniques qu'imprimés. Le CD-ROM s'avère, en ce sens, un support idéal pour plusieurs applications:

LES BASES DE DONNÉES, aussi bien bibliographiques (ex. ERIC, MEDLINE, NTIS, ABI/INFORM) que numériques (ex. SUPERMAP: US Census), factuelles (ex.: PC-SIG: collection de logiciels du domaine public) ou plein texte (ex. The BIBLE LIBRARY);

LES RÉPERTOIRES: bottins téléphoniques, listes d'institutions, listes de membres (ex. HOMEBASE et BUSINESSBASE de Tetragon; BusinessLine de Donnelly Marketing Information Services);

LES OUVRAGES DE RÉFÉRENCE: encyclopédies (ex. The ELECTRONIC ENCYCLOPEDIA de Grolier), almanachs, dictionnaires (ex. BOOKSHELF de Microsoft; le DICTIONNAIRE THÉMATIQUE VISUEL de Québec/Amérique/Facts on File), et autres (DISSERTATION ABSTRACTS de UMI; BOOKS IN PRINT PLUS de Bowker; DIRECTORY OF LIBRARY AND INFORMATION PROFESSIONALS de ALA, etc.);

LES INVENTAIRES D'ÉQUIPEMENTS, manuels d'entretien, etc. (ex. Boing, Renault); les CDROMs remplacent ici les microfiches;

L'ARCHIVAGE de rapports, journaux (ex. GLOBE AND MAIL; WALL STREET JOURNAL) et documents administratifs (ex. le GUIDE DE RENSEIGNEMENTS PERSONNELS du Conseil du Trésor du Canada); 
L'INFORMATION STATISTIQUE ET FACTUELLE: données d'enquêtes, séries chronologiques, données météorologiques, données économétriques, etc.;

LES CATALOGUES DE BIBLIOTHĖQUES, catalogues collectifs, catalogues commerciaux, etc. (ex. UTLAS, OCLC, FAXON, LISE de la Bibliothèque Publique d'Information en France et IBYCUS de I'Université de Columbia).

Plusieurs institutions ont déjà recours au CDROM pour leurs activités de stockage et de manipulation d'informations de toutes sortes, reflétant ainsi sa polyvalence d'application. Au Centre canadien de recherche sur l'informatisation du travail (CCRIT), par exemple, le CD-ROM est utilisé aussi bien comme support à des produits commerciaux (ouvrages de référence, bases de données) que comme support au catalogue et aux différents fichiers internes du Service intégré des Ressources d'information (SIRI). De plus en plus, des bibliothèques, centres de documentation, agences gouvernementales, compagnies, bureaux d'avocats et de médecins ainsi que des industries, exploitent les possibilités énormes du CD-ROM en matière de gestion de l'information.

En fait, chaque utilisateur actuel d'information peut devenir un usager du CD-ROM si ses besoins d'information rencontrent au moins les deux conditions suivantes: 1) s'il a un besoin fréquent de consultation d'un volume énorme de données; 2) si les informations pouvant être enregistrées sur CD-ROM ne nécessitent pas des mises à jour trop fréquentes ( 3 ou 4 fois par année).

\section{Le marché des technologies optiques}

\section{Caractéristiques}

Les caractéristiques des disques optiques leur accordent de nombreuses possibilités d'application dans les diverses couches du marché de l'information. Nous présentons dans cette section les structures de base des deux composantes de ce marché, soit le marché ouvert et le marché fermé. Cette distinction pourra être utile pour mieux cerner les opportunités de commercialisation des disques optiques.

\section{Le marché ouvert}

Le marché ouvert se distingue par le fait qu'il n'impose aucune condition d'accès et d'utilisation, sauf la volonté de payer le prix requis; il n'y a aucune barrière non économique dans ce type de marché. Le désir ou la capacité de payer le prix indiqué relève plutôt d'une décision personnelle ou organisationnelle, comme c'est le cas pour les autres décisions de nature économique dans une société capitaliste comme la nôtre.
La production de l'information (création, mise en mémoire, distribution et/ou recherche) dans le marché ouvert vise quatre sous-marchés qui sont: la consommation de masse, les affaires, les domaines de l'éducation et de la formation, des bibliothèques et enfin, celui de la recherche.

\section{Le marché de consommation de masse}

Les principales caractéristiques de cette couche de marché sont: une économie d'échelle due à une production et à une distribution de masse de produits d'information variés; une utilisation facile et conviviale (sans apprentissage long et complexe); des produits prêts à utiliser; un prix de vente des produits en deça de 1000 \$ (équipement et logiciels); des produits d'information à caractère instructif ou de loisir; une disponibilité des produits dans un format préparé à l'avance.

Ces caractéristiques sont conditionnelles à la pénétration du marché de la consommation de masse par les produits optiques. A ce jour, c'est surtout le $C D$-audio qui répond à ces critères, bien que d'autres produits, comme le CD-ROM, y réponde de plus en plus également. Toutefois, nous émettons encore certaines réserves quant à l'exploitation de CD-ROM comme produit de grande consommation. À notre avis, le transfert technologique des produits magnétiques (en ligne) aux produits optiques n'est pas encore tout à fait complété et les produits CD-ROM actuels s'adressent encore beaucoup aux marchés verticaux spécialisés. Ce n'est toutefois qu'une question de temps avant que les produits $C D-R O M$ ne deviennent consommation de masse (ex. BOOKSHELF de Microsoft, ELECTRONIC ENCY CLOPEDIA de Grolier).

\section{Le marché des affaires}

Le marché des affaires se distingue principalement de celui de la consommation de masse par le fait que: les produits d'information sont d'usage spécialisé plutôt que général; l'actualité, la précision et la pertinence de l'information sont capitales et influencent directement les prix (et donc la rentabilité) des produits; une mise à jour périodique de l'information est nécessaire; le prix est plus élevé pour chaque unité d'information; il y a plusieurs niveaux de service; l'accès en direct à de l'information en temps réel demeure quand même un besoin permanent; l'exclusivité de l'information peut être un facteur important.

Pour que les technologies optiques puissent pénétrer le marché des affaires, les produits d'information doivent être conçus pour des couches spécifiques du marché (clientèlescibles). La première vague de produits optiques visant le marché des affaires consiste en de grandes bases de données de référence sur $C D$ - 
ROM, par exemple, des données financières, économiques, des indicateurs de performance par pays, ainsi que certaines informations non accessibles en ligne (substances dangereuses, etc.).

\section{Le marché du domaine de l'éducation, de la formation et des bibliothèques}

Voyons les principales caractéristiques de ce marché: l'approvisionnement en information est lié aux processus d'apprentissage et de recherche; les institutions du secteur public jouent un rôle dominant dans l'acquisition et la diffusion de l'information (rôle d'intermédiaire); les clients potentiels s'attendent à avoir accès à l'information gratuitement sinon à faible coût; la presque totalité de l'information disponible est du domaine public; le support électronique représente une alternative en économie de temps, mais les usagers, bien souvent, ne peuvent ou ne veulent pas échanger les économies de temps et de travail pour l'achat d'information électronique; il y a généralement une approche axée sur la collaboration et la coopération pour la création de bases de données.

Pour que l'intégration des technologies optiques dans ce marché soit couronnée de succès, il faut que les produits aient des points positifs assez significatifs pour remplacer les produits d'information disponibles en ligne et provoquer la percée du marché par des produits non disponibles en ligne. Une autre condition de succès est que l'utilisation des produits ne requière peu ou pas d'expérience de recherche en ligne; il faut de plus que les prix soient assez abordables pour permettre aux individus et aux petites organisations de se procurer les produits sur le marché.

\section{Le marché de la recherche}

Ce sous-marché partage quelques caractéristiques avec ceux des affaires et de l'éducation. II est semblable au marché des affaires à l'exception que le pouvoir d'achat (ou la volonté d'achat) des chercheurs se situe à un seuil beaucoup plus bas que celui des gens d'affaires. Ceci est principalement dû au fait que, pour plusieurs chercheurs et institutions de recherche, il est possible ou de pratique commune de substituer une dépense en acquisition d'information par une somme de travail supplémentaire ou par un salaire d'étudiant. Par exemple, un chercheur (ou son étudiant) peut facilement passer plusieurs heures ou même plusieurs jours à consulter manuellement des ouvrages de référence (chaque jour équivalent à un déboursé de $80 \$$ s'il était payé $10 \$$ de l'heure) au lieu de dépenser de $40 \$$ à $70 \$$ pour une recherche en ligne.

En second lieu, le secteur de la recherche partage la caractéristique suivante avec le marché de l'éducation et des bibliothèques: plusieurs des produits d'information de ces deux marchés sont dispendieux (en argent et en temps) alors qu'ils ne génèrent qu'une faible valeur économique directe. D'autre part, les avantages économiques indirects à long terme résultant de l'accès à de l'information à jour dans le secteur de la recherche sont difficiles à quantifier et se justifient difficilement dans ce secteur qui souffre déjà d'un manque chronique de ressources.

\section{Le marché fermé}

Dans les marchés dits fermés, l'accès et l'utilisation de l'information sont régis par des critères non économiques. Le pouvoir d'achat ne garantit pas automatiquement l'accès à l'information. Les marchés sont de deux types: les groupes fermés d'utilisateurs et les marchés internes.

\section{Les groupes fermés d'utilisateurs}

L'admission dans un groupe fermé d'utilisateurs et le droit d'accès aux sources d'information (bases de données ou réseaux) sont régis selon des critères définis par le groupe. De tels groupes comprennent, par exemple, les systèmes d'information et de transfert électronique de fonds d'institutions financières comme SWIFT; les chercheurs et organismes membres d'ARPANET; les bibliothécaires membres d'ALANET; les bibliothèques et centres de documentation échangeant des données de catalogage par OCLC ou UTLAS; etc.

L'information échangée au sein de ces groupes revêt généralement une grande valeur et une certaine confidentialité nécessitant des mesures de sécurité assez élaborées en termes de codage. II en résulte que les besoins (et la capacité de payer) de ces groupes sont des facteurs importants pour la stimulation du développement de supports électroniques d'information et de mécanismes de distribution.

\section{Les marchés internes}

Les opérations propres à cette couche du marché de l'information demeurent pour la plupart internes à l'institution. Ce sont surtout des applications d'archivage. Lorsque l'institution est assez grande, les besoins d'information (bases de données ou réseaux d'information) sont importants. Que les informations portent sur des données d'inventaires, des catalogues de produits, des fiches de clients, etc., les marchés internes ont un certain nombre de caractéristiques communes dont:

- un volume important d'information sur support électronique, souvent complété par des microformes; 


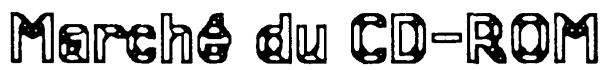

(Prévisions 1990)

$\begin{array}{ll}\text { Distribution de bases de données } & 34 \% \\ \text { Distribution de logiciels } & : 20 \% \\ \text { Applications didactiques } & : 15 \% \\ \text { Information légale/juridique } & : 7 \% \\ \text { Information médicale } & : 68 \\ \text { Point de vente } & : 5 \% \\ \text { Publication de périodiques } & : 48 \\ \text { Banques d'images } & : 38 \\ \text { Autres } & : 48\end{array}$

\section{Source: Rotchild Consultants}

\section{Fig. 6: Distribution des applications du CD-ROM}

un grand nombre d'unités dispersées en plusieurs lieux dans l'organisation devant avoir accès à la même information en même temps;

des systèmes électroniques d'information reliés ou constituant un sous-ensemble du réseau d'opérations et de transactions de l'organisation;

de nombreuses portes d'accès à l'information pour les usagers;

la nécessité de conservation des données (archivage);

- un cycle de vie assez long (au-delà de 3 mois) pour une bonne partie de l'information (documents actifs);

- un calendrier de conservation;

- des mesures de sécurité adéquates pour préserver la confidentialité et la valeur stratégique de l'information.
Actuellement, plusieurs organismes des secteurs public et privé ont des activités de production et de diffusion d'information interne sur des supports imprimés et électroniques ou sur microsupports. Cette situation représente une bonne opportunité de marché pour les technologies optiques. II ne faut pas non plus négliger I'utilisation de ces technologies pour la formation du personnel. Ce sous-secteur possède d'ailleurs une croissance constante. II en va de même pour la distribution de logiciels et diverses autres applications plus spécialisées, comme en fait foi la figure 6 présentant des prévisions (par Rotchild Consultants) de distribution, en 1990, des diverses applications du CD-ROM.

Selon Julie B. Schwerin d'Info-Tech, la grande «papesse» des mémoires optiques, un bon produit pour le CD-ROM est un produit de nature assez générale, qui est ou peut être converti en produit lisible par ordinateur et qui compte au moins 100 utilisateurs ${ }^{9}$. II s'ensuit de cette définition qu'une 


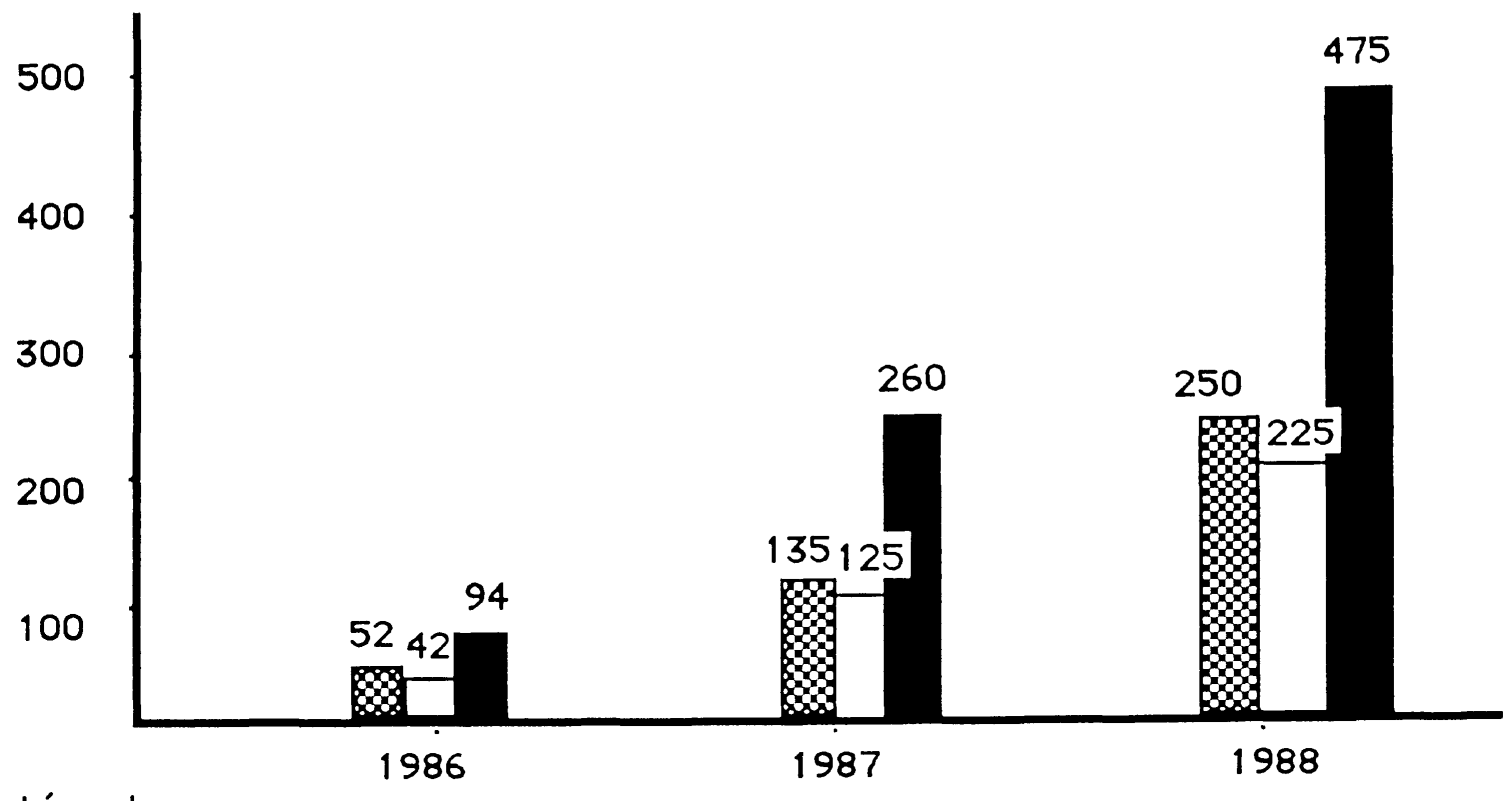

Légende

88 : commercial

: privé

: total quantité considérable de produits peuvent se qualifier pour une conversion ou une saisie sur CD-ROM, depuis l'annuaire téléphonique jusqu'au texte complet de magazines et de périodiques. II s'ensuit également que le marché du CD-ROM est pratiquement ouvert à toute personne ou à tout organisme utilisant de larges quantités d'information pouvant être digitalisée.

\section{Indicateurs économiques}

Schwerin et la compagnie Info-Tech tiennent un registre des principaux indicateurs économiques du marché des disques optiques. Les baromètres importants de ce marché, selon elles, sont les suivants ${ }^{10}$ :

\section{Marché primaire}

Baromètre no 1: nombre de titres commerciaux disponibles;

Baromètre no 2: nombre de lecteurs de disques optiques installée;
Baromètre no 3: nombre de produits et disques CD-ROM vendus;

Baromètre no 4: valeur totale des ventes aux utilisateurs;

\section{Marché secondaire}

Baromètre no 5: dépenses en publicité des divers intervenants de l'industrie du disque optique;

Baromètre no 6: structure de l'industrie du $C D$ ROM et provenances des intervenants.

À cette liste, on pourrait également ajouter le nombre et le type de points de vente des produits et des équipements CD-ROM. Par exemple, au Québec, il faut encore s'adresser directement au manufacturier pour acheter des produits $C D$ ROMs (disques, équipements). Cette situation devrait changer bientôt puisque Tandy Corporation vient d'annoncer une entente avec Hitachi pour distribuer ses équipements CD-ROMs dans les boutiques de Radio-Shack. Cela devrait "ouvrir» grandement le marché des CD-ROM au Québec et engendrer plusieurs effets d'entraînement (autres ententes, distribution de produits, etc.).

10. Julie B. Schwerin, «Introduction to the optical disc forum Ilw, Electronic and Optical Publishing Review, vol. 7, no. 4 (December 1987), 160-168. 


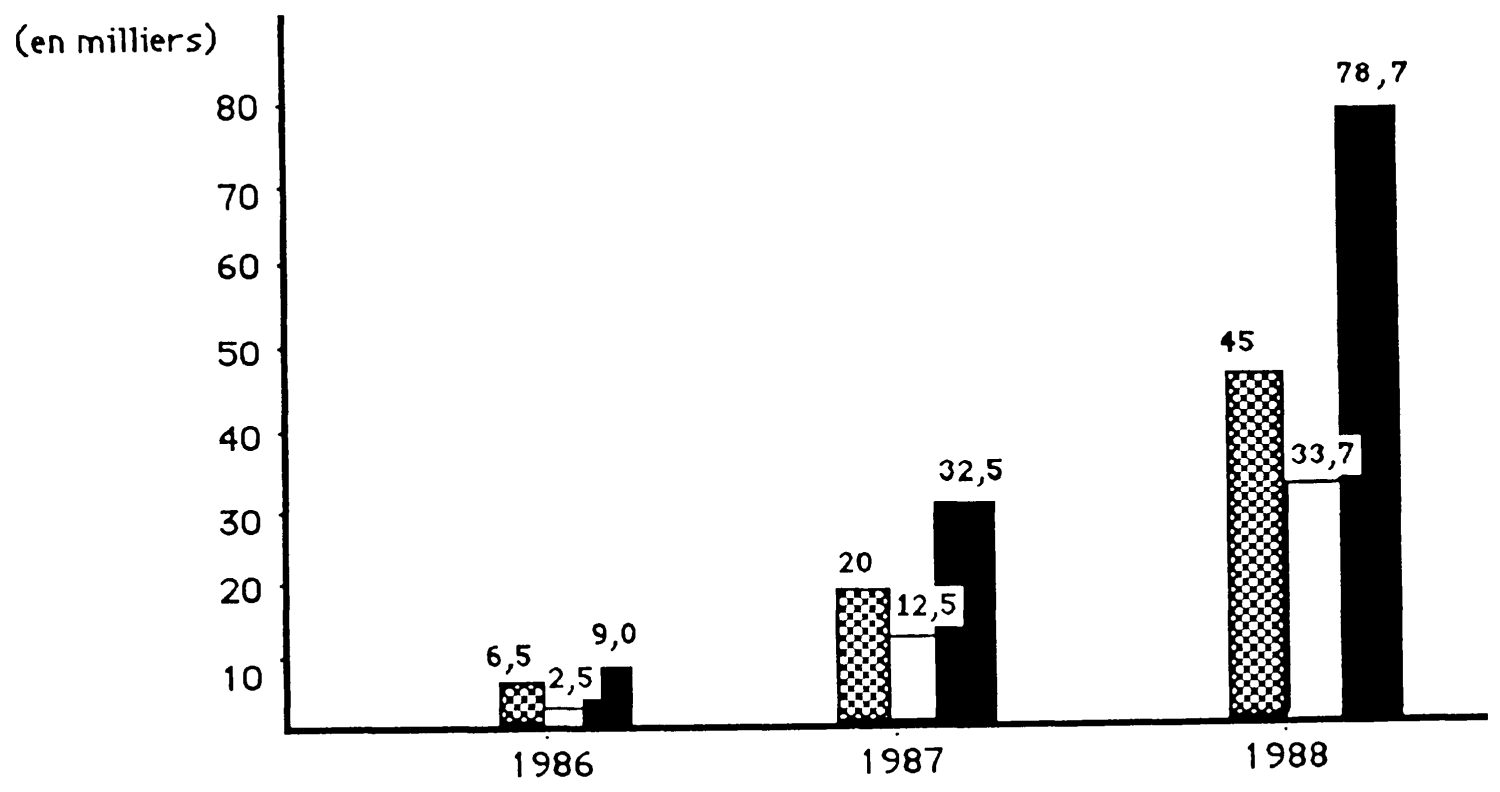

Légende

\section{:commercial $\square$ :privé : total}

\section{Fig. 8 : Titres de bases de données: copies vendues}

À partir de ces indicateurs, Info-Tech compile et tient à jour des statistiques sur le marché des disques optiques, ce qui permet de suivre plus facilement l'évolution de ce jeune marché. Ainsi, pour les deux premiers baromètres - nombre de titres de bases de données et nombre de lecteurs -, Info-Tech a compilé les chiffres indiqués aux figures 7 à $12^{11}$.

Comme on peut le constater dans ces figures, le marché du disque optique est en croissance exponentielle: sur presque tous les indicateurs retenus, il montre une augmentation de $100 \%$ entre 1986 et 1987 et encore entre 1987 et 1988 . C'est le signe d'un marché jeune et en pleine explosion.

Les études de marché, même si elles divergent parfois quant à sa vitesse de croissance et à l'importance de son potentiel éventuel, sont unanimes à montrer une industrie encore très nouvelle, en pleine effervescence et au potentiel énorme. On estime à l'heure actuelle que la demande pour les produits optiques est plus rapide que celle de n'importe quel autre support d'information. Même la téléréférence, à ses débuts, n'a pas connu une croissance aussi accélérée. C'est peut-être également ce qui explique la disparité des chiffres et des prévisions des diverses études de marché, comme en témoigne le tableau synoptique représenté à la figure $13^{12}$

D'autre part, une étude de marché de Electronic Trend Publications, de Saratoga en Californie, montre qu'en 1987, la part des technologies optiques dans le marché total du stockage de l'information, était estimée à 1698 millions \$ US,
11 Ibid., 167.
12. FS : Frost and Sullivan, Unix World (January 1987), 32.

FA : Freeman and Associates, Unix World (January 1987). 32.

ERC Evans Research Corporation, EDP In-Depth Report. vol. 15, no. 9 (September 1986), 13.

PCW: PC Week (April 1987), 220-259

IT : Info-Tech, Julie B. Schwerin, alntroduction to the optical disc forum II...*

IRD International Resources Development, Online, vol 12, no. 3 (May 1988), 90-91.

LCR : Link Resources Corporations, 10th International Online Meeting, London 2-4 Dec. 1986, p. 263-269. 


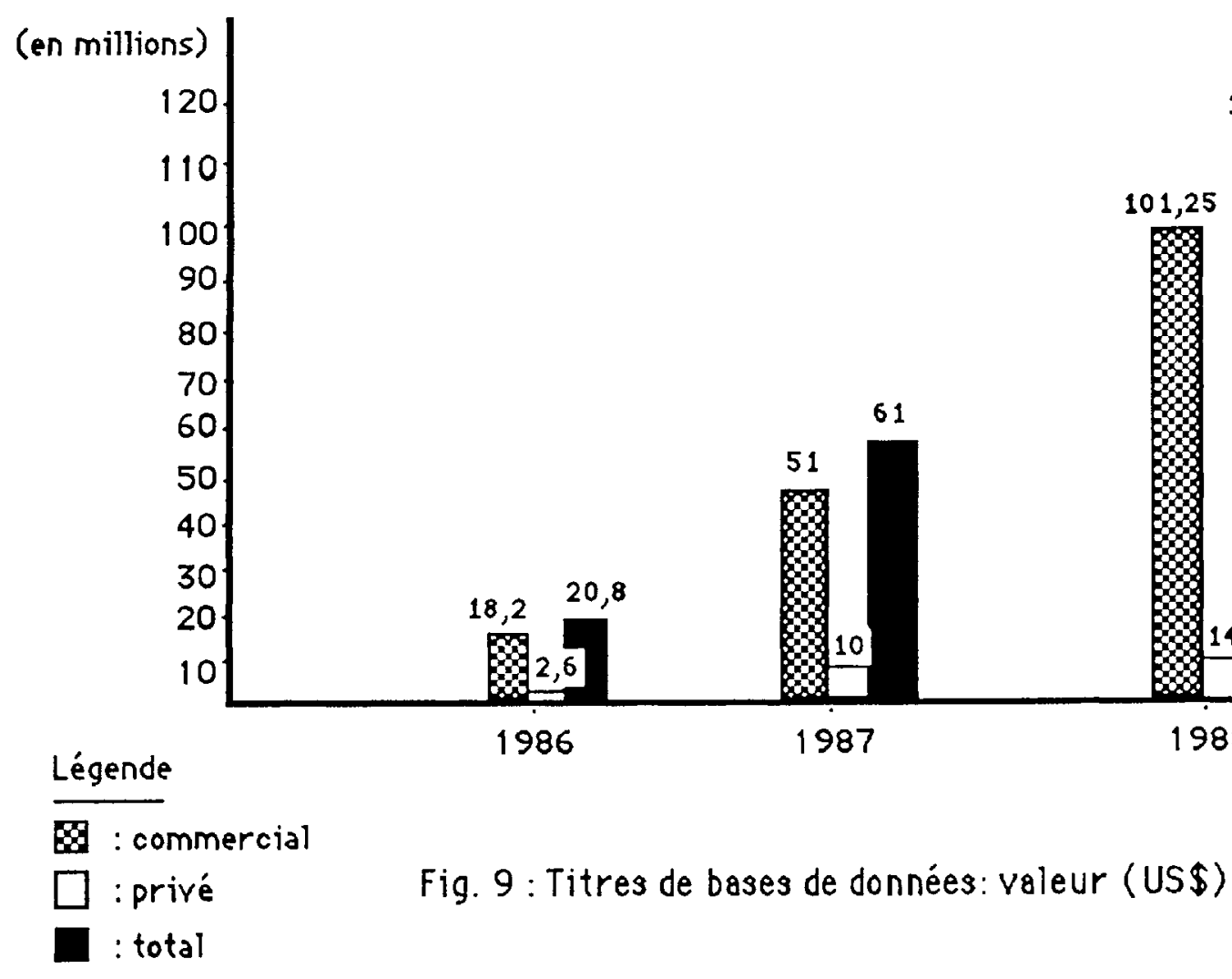

(en milliers)

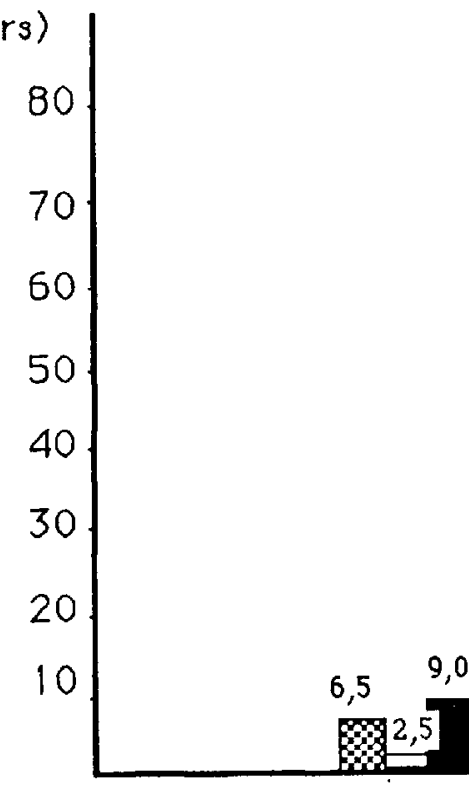

Légende

1986

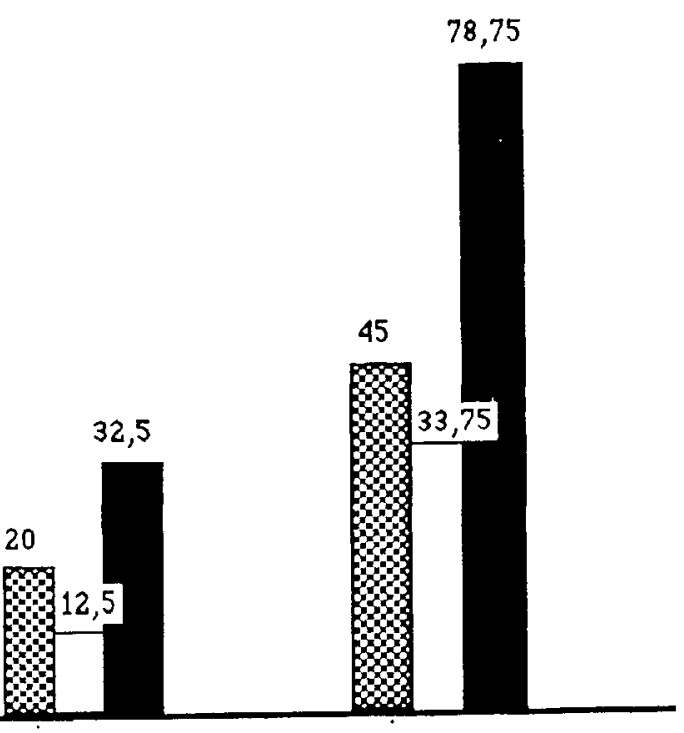

1987

1988

8. commercial

$\square$ : privé

Fig. 10 : Lecteurs de disques optiques: nombre de lecteurs instollés

: 1002 
(en milliers)

Légende

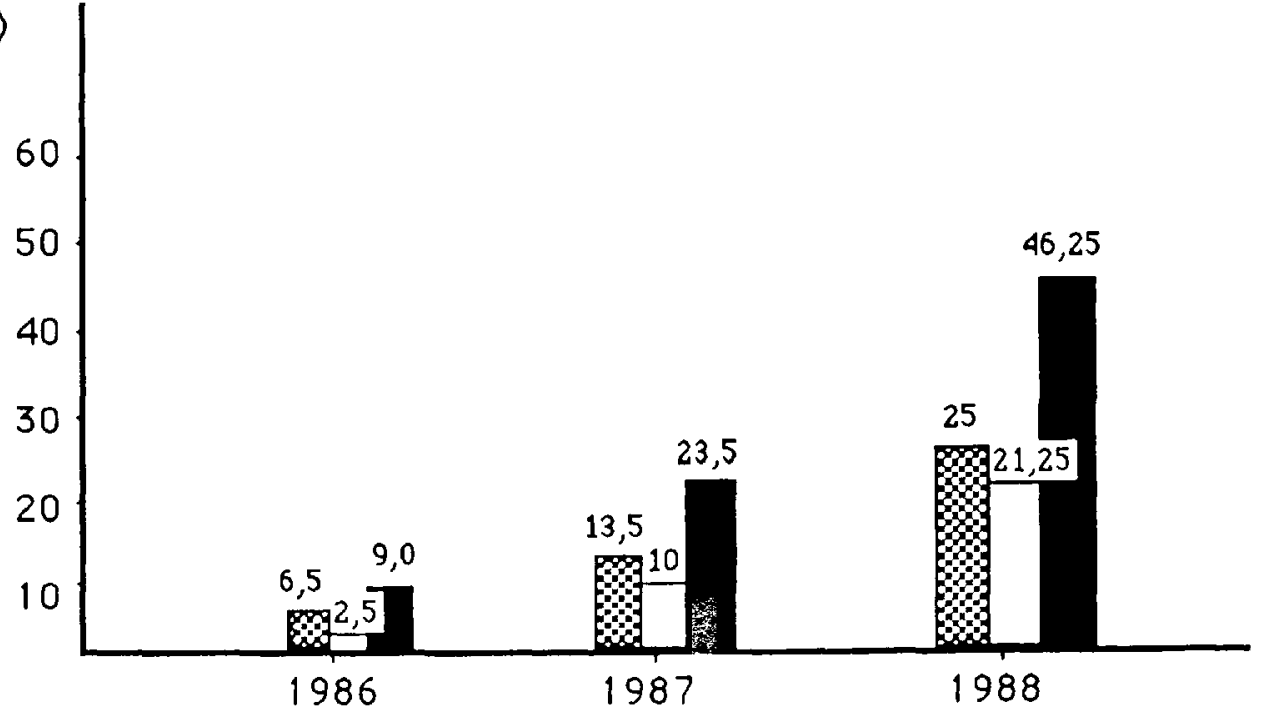

B8: commercial

$\square$ : privé

Fig. 11 : Lecteurs de disques optiques: nombre de lecteurs vendus à date

(en millions)

Légende

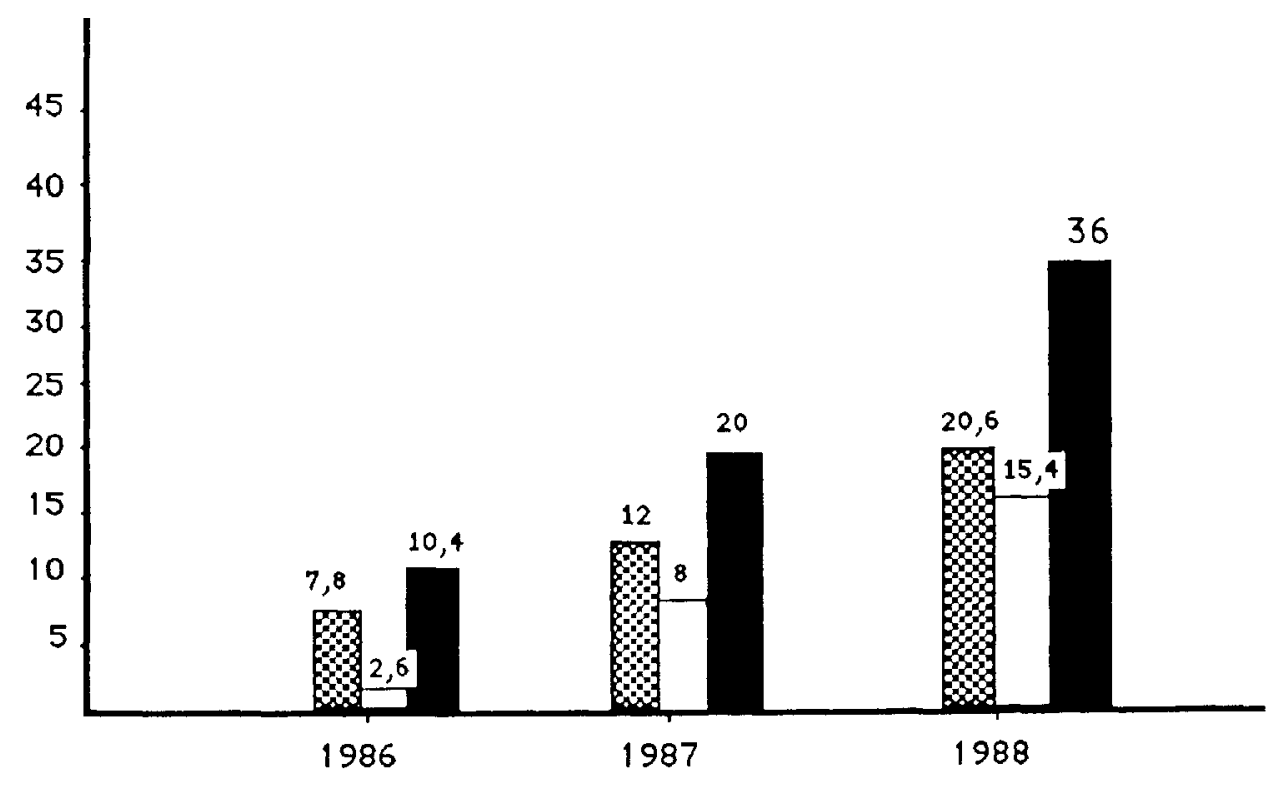

B : commercial

$\square$ : privé

Fig. 12 : Lecteurs de disques optiques: valeur totale (US $\$$ )

: total 


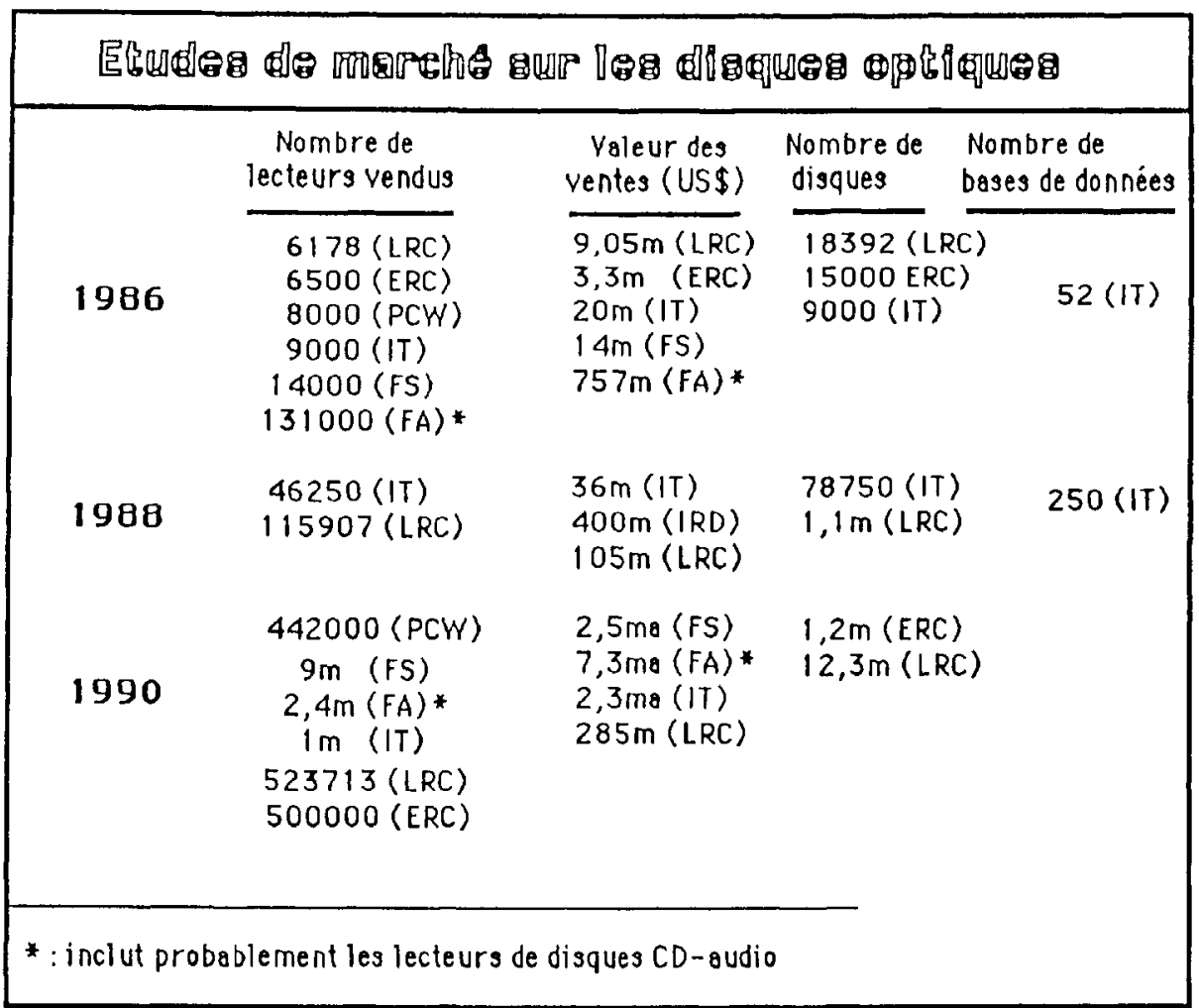

Fig. 13 : Typologie des études de marché sur les disques optiques

\begin{tabular}{|l|l|}
\hline \\
$\$ 1987$
\end{tabular}

Fig. 14 : Distribution des supports de stockage 
soit à peine $0,8 \%$ du marché. En 1991, on prévoit un marché de 17889 millions \$ US, soit une part de $4,7 \%$ du marché. La croissance prévue serait donc de facteur 10 en 4 ans seulement ${ }^{13}$.

Une étude de Frost and Sullivan, d'autre part, montre que l'industrie du CD-ROM n'a pas tout à fait connu la croissance prévue ${ }^{14}$. Les raisons avancées pour expliquer ce phénomène sont: l'absence de réversibilité des supports optiques; l'accès plus lent que celui des disques Winchester; l'absence des standards; l'amélioration technique des supports magnétiques (ex. disques durs sur cassettes).

L'étude prévoit tout de même une croissance annuelle de $80 \%$ en valeur pour les unités de stockage optique. II n'en demeure pas moins que la croissance soudaine et rapide de ce marché ouvert et très compétitif a entraîné de nombreux flous et notamment des problèmes de tarification pour les producteurs. Selon Patrick Gibbins ${ }^{15}$, jusqu'à ce que l'industrie du CD-ROM n'atteigne un certain niveau de maturité, avec un parc de lecteurs optiques dans les millions d'unités (plutôt que dans les milliers) et avec des ventes de produits dans les milliers d'unités (plutôt que dans les centaines), les éditeurs et producteurs de bases de données et les concepteurs de logiciels continueront d'avoir de la difficulté à déterminer une structure de prix de leurs produits. L'objectif, à court terme, doit être d'accroître la masse critique de produits disponibles au consommateur, d'établir et de consolider la confiance dans le CD-ROM comme support d'édition électronique par excellence pour au moins les 10 prochaines années.

\section{Marché des bibliothèques}

Les producteurs d'information sur CD-ROMs destinés au marché des affaires et au secteur de la recherche (produits de 2000 s et plus), furent surpris de découvrir qu'un nombre significatif de leurs premiers acheteurs provenaient du secteur de l'éducation et des bibliothèques. Apparemment, les bibliothèques ont déjà identifié, dans les CD-ROMs, plusieurs avantages par rapport à l'imprimé et à l'interrogation en ligne. Les principales raisons de cette préférence des bibliothèques pour les CD-ROMs, selon les auteurs, seraient:
- la possibilité de retourner à l'approche plus traditionnelle axée sur l'offre de l'information plutôt que l'approche en-ligne, axée sur la demande;

une gestion plus facile des coûts administratifs: le mode d'interrogation en ligne ne garantit pas le paiement immédiat du temps de recherche; le non-paiement ou les retards ralentissent la rentrée des revenus;

- une plus grande stabilité pour budgétiser et définir les allocations d'usage; un CD-ROM représente un seul coût fixe (comme une monographie ou un abonnement de périodique) alors que l'interrogation en ligne implique des coûts variables plus difficiles à contrôler et à prévoir;

une certaine socialisation des produits documentaires: les produits d'information acquis par des fonds publics sont utilisés et réutilisés par tous, alors que les résultats de recherches en ligne répondent au profil de chaque utilisateur et ne sont donc pas utiles à plusieurs;

- la facilité d'utilisation: Ie CD-ROM accorde plus d'autonomie à l'usager, ce qui économise des frais d'intervention en personnel (notamment pour les connexions et les recherches).

Aujourd'hui, selon Julie B. Schwerin ${ }^{16}$. environ $50 \%$ du marché du CD-ROM sont occupés par les bibliothèques, les autres $50 \%$ étant les plus gros utilisateurs de bases de données en ligne tels les compagnies et les gens d'affaires, les firmes d'avocats et les organismes techniques spécialisés. Le marché pourrait devenir aussi vaste que celui proposé dans la définition ${ }^{17}$, mais plusieurs étapes seront encore nécessaires avant qu'il n'atteigne ce potentiel.

Une analyse de 164 titres différents de bases de données listées dans le numéro de décembre 1987 de CD-ROM Review ${ }^{18}$, montrait la typologie de ces bases (Voir figure 15).

La très grande majorité de ces bases de données s'adresse directement au marché des bibliothèques.
13. Gene Selven et Michael Moon, «Long awaited bonanza in optical storage finally arrives», Laserdisk Professional, vol. 1 , no. 1 (May 1988), 86-89.

14. Infotecture, no 158 (5 mai 1988), 4

15. Patrick Gibbins, «Pricing software and information on CDROMm, Electronic and Optical Publishing Review, vol. 7. no. 4 (December 1987), 176-180
16. Julie B. Schwerin, «Introduction to the Optical Disc Forum II....

17. Ibid

18. Janet M. Tiampo, "CD-ROM disc titles", CD-ROM Review, vol. 2, no. 5 (Nov./Dec. 1987), 48-55. 
TEXTUELLES

BIBLIOGRAPHIQUES

CATALOGRAPHIQUES

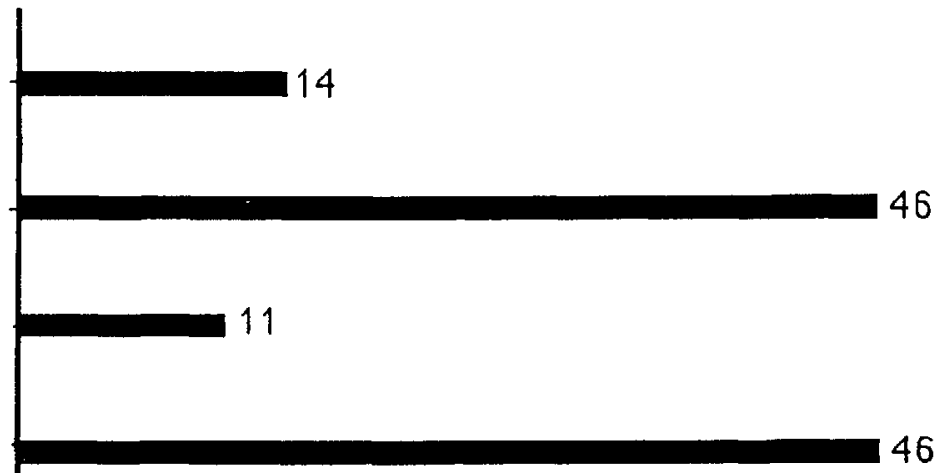

STATISTIQUESI NUMERIQUES

AUTRES

$(N=164)$

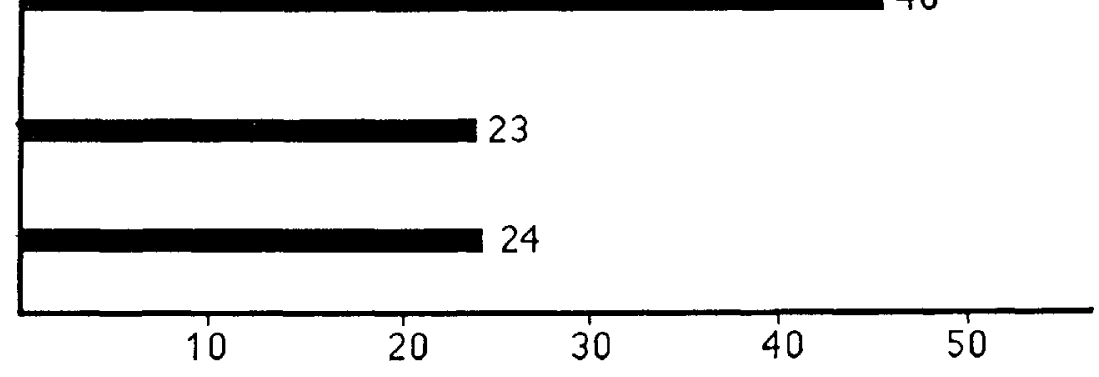

Fig. 15 : Typologie des bases de données sur CD-ROM

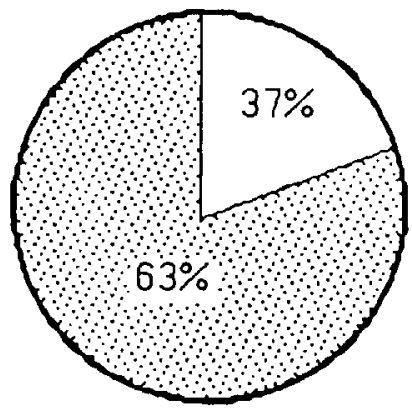

1985

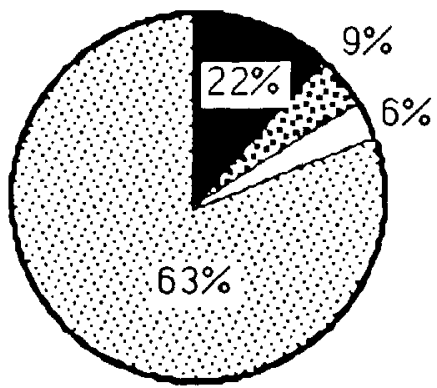

1990

: Professionnel

$\square$ : Bibliothèques

- Education

: Grand public

Fig. 16 : Répartition des marchés d'application du CD-ROM (Link et Infotech) 
Pour sa part, Véronique Charreyron ${ }^{19}$ cite la répartition suivante des marchés d'application du CD-ROM, proposée par Link et Info-Tech (1986): Professionnel, bibliothèques, éducation, grand public (Voir figure 16).

Ces prédictions assez pessimistes de l'évolution du marché des bibliothèques pour les CD-ROM ne sont toutefois pas partagées par tout le monde. R.J. Rietdyk, vice-président de Silver Platter Inc., estime qu'il y a un marché de plus de 40000 bibliothèques aux États-Unis et au Canada et de plus de 100000 autres pour le reste du monde. Selon lui, les producteurs de bases de données sur CD-ROM, pour atteindre le marché des bibliothèques, doivent en connaître les principales caractéristiques ${ }^{20}$ :

1. Marché bien informé et bien structuré;

2. Marché de professionnels compétents, formés à la recherche de l'information;

3. Marché qui désire l'information la plus complète et la plus à jour possible;

4. Marché de décideurs aux prises avec un processus de décision lent et complexe;

5. Marché d'institutions fonctionnant avec des budgets limités;

6. Marché d'institutions fonctionnant avec un cycle budgétaire annuel;

7. Marché d'institutions généralement fidèles à leurs fournisseurs.

Par ailleurs, deux études récentes présentent également une image plus optimiste du marché des bibliothèques pour le CD-ROM. La première est une enquête de la firme Research Publications, à l'été 1987, auprès des bibliothécaires et spécialistes de l'information des Etats-Unis et du Canada $^{21}$. Sur les 20443 répondants, 2956 $(14,4 \%)$ utilisaient déjà (au moins) un produit CDROM, 1903 (9,3\%) prévoyaient en utiliser un avant la fin de 1987, 2605 (12,7\%) avant la fin de 1988, 1233 (6\%) avant 1989 et $1471(7,2 \%)$ avant la fin de 1990. Par conséquent, 10168 répondants utiliseraient un produit CD-ROM avant la fin de 1990, soit environ 50\% du marché de la documentation. Les plus gros utilisateurs sont les bibliothèques universitaires qui passeraient de 2523 en 1987 à 4661 en 1990 .

La seconde étude est une enquête téléphonique menée par la firme Saperstein Associates pour OCLC Inc., en 1987, auprès de 301 de ses membres et de 150 non-membres ${ }^{22}$. Les résultats montrent qu'en 1986, $9 \%$ des répondants $(6 \%=$ membres, $3 \%=$ non-membres $)$ possé daient des produits CD-ROMs et qu'en 1987, la proportion était passée à $31 \%(27 \%=$ membres, $4 \%=$ non-membres), pour un taux de croissance de l'ordre de $344 \%$. Ici encore, les bibliothèques universitaires s'avéraient, en 1987, les plus grosses utilisatrices (62\%) comparativement aux autres types de bibliothèques (20\%). Les principales raisons avancées par les répondants pour justifier l'achat de produits CD-ROMs sont: la réduction des coûts d'interrogation en ligne (23\%), la grande capacité de stockage (17\%), la meilleure option $(17 \%)$ et l'accès facile (16\%).

La première raison est particulièrement intéressante. Le transfert technologique des produits en ligne aux produits optiques force la comparaison, comme il en avait été lors du passage de l'imprimé au support informatique. C'est d'ailleurs ce que nous analyserons plus en détail dans la seconde partie de cet article. Mentionnons simplement qu'à l'instar de l'imprimé et malgré certaines dénégations, le marché de la téléréférence réagit à l'apparition du disque optique. Les serveurs se sentent à juste titre menacés et certains (Dialog, BRS, Wilson) se sont lancés à l'assaut du marché. Le service d'interface EasyNet a même annoncé ${ }^{23}$ des coûts fixes annuels d'interrogation en ligne avec sa "Answer Machine»; I'un des plans proposés est un coût fixe annuel de $30000 \$$ US à 35000 S US pour un accès illimité à toutes les bases de données de tous les serveurs auxquels il offre l'accès.

\section{Rôle des bibliothèques}

Quel est le rôle des bibliothèques face à ce nouveau produit? Deux options s'offrent à elle: un rôle de consommation et un rôle de production.

\section{Rôle de consommation}

Le rôle de consommation des bibliothèques consiste à évaluer, à acheter et à offrir à ses clientèles les meilleurs produits sur le marché susceptibles de satisfaire leurs besoins et d'aider ses clientèles à bien les exploiter. C'est le rôle conventionnel de la bibliothèque qu'elle joue déjà très bien avec son service de référence et de téléréférence. D'ailleurs la majorité des produits
19. Véronique Charreyron, «Le CD-ROM et ses premières applications: 1988, l'année de tous les espoirs", L'ordinateur individuel, no 101 (mars 1988), 92-96.

20. R.J. Rietdyk, "Creation and distribution of CD-ROM databases for the library reference desk", Journal of the American Society for Information Science, vol. 39, no. 1 (January 1988), 58-62.

21. Voir publicité dans Online, vol. 12, no. 3 (May 1988), 90 91 et dans Information Hotline, (March 1988), 3.
22. "OCLC compact disk study", Laserdisk Professional, vol. 1, no. 1 (May 1988), 44-49.

23. "EasyNet's revolutionnary answer machine targets public and academic libraries; Threatens price appeal of CD-ROM", Database searcher, vol. 3, no. 8 (September 1987), 20. 
CD-ROMs disponibles commercialement pour les bibliothèques sont de produits de référence (index et abstracts, encyclopédies, dictionnaires, répertoires, etc.).

Parmi les facteurs que la bibliothèque doit considérer avant de faire l'acquisition des produits CD-ROMs, on retrouve:

- I'accès et la disponibilité: nombre/complexité des recherches en ligne faites dans une base de données spécifique; disponibilité et coût du CD-ROM (équipement et bases de données); durée de vie des données recherchées (information ponctuelle, etc.); fréquence des mises à jour; désir d'autonomie de la part des usagers et leur niveau de connaissance des techniques de recherche;

- le personnel diponible: temps/personne requis pour assister les usagers (recherches complémentaires nécessaires); formation des usagers par le personnel; disponibilité d'un manuel d'opération;

- les coûts: coûts défrayés pour la recherche: réallocation du budget des recherches en ligne ou du budget de référence (ouvrages imprimés) à l'achat de CD-ROMs; équipement: nombre de postes de travail, coûts d'installation; bases de données: coûts d'abonnement, coûts des lecteurs.

Un projet-pilote avec un lecteur et une base de données (la plus populaire) ou un ouvrage de référence (encyclopédie, dictionnaire souvent utilisés) permettra de prendre le pouls des clientèles pour ce nouveau produit, de développer des méthodes et des outils de formation des clientèles, d'observer leur comportement lors des interrogations, de mettre sur pied un service de dépannage et d'établir des comparaisons et peutêtre même des liens de service avec la référence «manuelle» et la téléréférence, sans compter la visibilité accrue de la bibliothèque.

Selon Howard Silver, de la Hahnemann University Library de Philadelphie ${ }^{24}$, les principales connaissances et compétences requises de la part du personnel de la bibliothèque (référence, circulation) pour assister les utilisateurs de disques sont la compétence dans la manipulation des équipements (comment installer et changer un disque CD-ROM; comment ouvrir et fermer tous les appareils (micro-ordinateur, lecteur, imprimante); comment ajouter du papier et dégager les imprimantes lorsque le papier est bloqué; comment changer les rubans des imprimantes; comment redémarrer une machine bloquée; connaissance du clavier du micro-ordinateur) et la connaissance des logiciels d'exploitation et des logiciels d'application (comment installer un logiciel; comment débuter et terminer une session; stratégie élémentaire de recherche et utilisation des opérateurs logiques; recherche sur un champ spécifique; visualisation et impression d'informations; télédéchargement d'informations du disque optique à la mémoire du microordinateur; procédure pour la formation des nouveaux utilisateurs; interruption des messages d'erreur et dépannage simple).

\section{Rôle de production}

La bibliothèque peut également envisager produire certaines informations ou fichiers sur CDROM. C'est le cas, de toute évidence, avec les catalogues (monographies, périodiques, etc.). Certains systèmes existent déjà commercialement qui permettent à la bibliothèque: 1) d'acheter des catalogues de grandes bibliothèques (BIBLIOFILE) ou des catalogues collectifs (OCLC, UTLAS) et 2) de produire son propre catalogue sur CD-ROM (Auto-Graphics, Brodart, Library Corporation, LSSI/OCS, Marcive, UTLAS, etc. $)^{25}$. Ce pourrait être le cas également avec certains fichiers plus spécialisés, certaines collections éphémères de recherche et même, certains programmes et outils de promotion et de formation des clientèles.

Toutefois, un certain nombre de conditions doivent être satisfaites pour que cette production soit «rentable» pour la bibliothèque:

Le contenu des bases de données ou des fichiers à enregistrer sur CD-ROM doit être original et d'intérêt assuré; la bibliothèque ne devrait en aucun cas tenter de compétitionner avec les producteurs commerciaux;

- Certaines caractéristiques de distribution doivent être prises en considération: le nombre d'usagers à l'interne, le nombre de sites à desservir (postes de travail) et le nombre de clients externes potentiels (autres bibliothèques, organismes) vont déterminer le nombre de copies à tirer de chaque produit. On avance surtout le seuil minimal de 100 copies pour atteindre une certaine «rentabilité»;
24. Howard Silver, "Managing a CD-ROM installation. A case study at Hahnemann University», Online, vol. 12, no. 2 (March 1988), 61-66.
25. Voir à cet effet l'excellent article de Linda W. Helgerson, «Acquiring a CD-ROM public access catalog system. Part 1: The bottom line may not be the top priority", Library Hi-Tech, vol. 5, no. 3 (Fall 1987), 49-75. 
- La fréquence de mise à jour ne devrait pas être trop grande; les mises à jour trimestrielles sont les plus répandues pour les disques optiques; pour une mise à jour plus fréquente de son catalogue, la bibliothèque devra donc envisager des solutions intermédiaires (en ligne, listes imprimées, microfiches);

Le volume d'information doit être suffisamment important pour justifier son stockage sur disque optique. Seuls les catalogues des grandes bibliothèques répondent à ce critère; ou alors, il faut privilégier des programmes de publication conjointe. Par exemple, le partage d'un disque optique entre plusieurs petites bibliothèques ou centres de documentation pour leurs catalogues respectifs ou mieux, si possible, pour des catalogues collectifs;

- Les modes d'accès et d'interrogation doivent être simples et conviviaux. Cela peut signifier, par exemple, la création d'un logiciel de recherche ou la modification d'un logiciel existant.

\section{Conclusion}

Dans cette première partie, nous avons voulu présenter aux bibliothécaires et spécialistes de la documentation, ce nouveau support d'information qu'est le disque optique et plus spécifiquement le CD-ROM. Nous avons brièvement présenté ses caractéristiques techniques, ses avantages et ses inconvénients. Nous avons également analysé le marché des technologies optiques, son état actuel, son évolution et son avenir. Déjà on peut dire qu'il a passé le stade de l'enfance et qu'il entre maintenant dans l'adolescence. À ce jour, les technologies et produits optiques (spécialement les CD-audio et les CD-ROMs) disponibles sur le marché se situent parmi les supports les plus fiables et résistants pour la conservation de l'information numérisée.

Leurs caractéristiques de compacité et de duplication permettent de les poster à peu de frais partout dans le monde en de nombreux exemplaires pour un coût marginal minime. Un réseau parallèle à celui offert par les serveurs (bases de données en ligne) peut ainsi être créé tout en offrant aux usagers une certaine autonomie d'action. Cependant, comme pour le choix de tout support d'information, il convient de bien connaître ses besoins afin d'exploiter pleinement le potentiel des disques optiques. Une comparaison avec les coûts et le rendement d'autres alternatives (support papier, abonnement à un serveur en ligne, microfichage, etc.) est souvent utile. Nous aborderons cette question de l'évaluation (descriptive, comparative, expérimentale) dans la seconde partie de cet article. Le processus de production des CD-ROMs repose sur une technologie bien établie et acceptée mondialement par l'industrie. La question des normes (High Sierra, ISO, SCSI) qui a longtemps ralenti la croissance de l'industrie, est à toutes fins pratiques réglée. Cependant, les méthodes d'indexation et d'interrogation ainsi que l'organisation des menus et programmes d'aide à l'usager sont encore sujettes à plusieurs variations de la part des éditeurs. Cette étape d'organisation des données avec le logiciel d'accès et de recherche est appelée à subir plusieurs modifications pour en arriver, si possible dans le futur, à une normalisation des méthodes utilisées, ou du moins, à une entente sur les pratiques généralement acceptées par l'industrie. Nous ne sommes toutefois pas débordants d'optimisme sur cette question..

II est à noter que les coûts de production d'un CD-ROM dépendent directement de la qualité d'organisation des données originales, c'est-àdire du degré de préparation requis pour qu'elles soient interrogeables sur CD-ROM. Cette phase de production se révèle en effet la plus longue et la plus dispendieuse, alors que la phase de duplication (production des copies) se fait à un coût relativement bas; celui-ci baissant proportionnellement avec l'augmentation du nombre de copies produites et avec un coût unitaire plus faible, il est possible de faire davantage de copies.

Bien que le nombre d'ouvrages édités sur CDROM soit encore assez limité (environ 250 à 300), la variété des produits disponibles exprime de façon significative le large éventail des applications possibles, chacune constituant de réelles ouvertures de marché. Jusqu'à l'avènement du CD-ROM, la distribution électronique de l'information nécessitait des investissements considérables incluant l'équipement (mini et gros ordinateurs), des logiciels d'interrogation dispendieux, une forte capacité de mémoire et l'établissement ou la location de réseaux de télécommunication. Les coûts de fonctionnement étaient également élevés en termes de documentation pour les usagers, leur formation, le support (dépannage) et les services d'information de même que la gestion (monitoring) des activités des usagers, incluant le contrôle des mots de passe, et la collecte des revenus.

Il est vrai que par l'utilisation commune de serveurs tels Dialog, Can/Ole, QL Systems, IST Informathèque, etc., des économies d'échelle peuvent être atteintes, de même qu'une certaine optimisation des opérations. Mais le fait est que les dépenses de base sont élevées. De plus, il est nécessaire d'accroître les investissements en capital (plus gros ordinateur, etc.) pour servir un nombre croissant d'usagers. 
Il est admis que la production d'un CD-ROM à partir de bases de données peut coûter de $4000 \$$ à $25000 \$$. Mais un CD-ROM peut être produit trimestriellement pendant 3 ans à un moindre coût que celui de l'établissement d'un réseau en ligne. De plus, la distribution de l'information au moyen du CD-ROM amène un transfert de la tâche de l'investissement en capital, du vendeur à l'acheteur d'information (ce dernier ayant à acheter le lecteur optique).

Une fois le disque-maître produit, il n'y a pratiquement pas de limite au nombre de copies pouvant être faites. Ainsi, un réseau de plusieurs millions d'usagers «en ligne» peut être supporté à un très faible coût marginal et à des niveaux élevés de profit (ou de récupération des coûts). Il doit être aussi noté que l'utilité d'un CD-ROM excède celles du papier et des services en ligne dû aux algorithmes de recherche des logiciels d'interrogation (recherches multicritères) et à la liberté d'action de l'usager qui n'a pas à se préoccuper des frais de connexion et de télécommunications.

Les services d'information en ligne (ex. I.P. Sharp, Reuter) offrant l'accès à des données éphémères (données financières, actualités) nécessitant de fréquentes mises à jour, ont peu de concurrence à craindre de la part des produits CD-ROMs. En fait, les fournisseurs actuels de services d'information en ligne peuvent trouver des avantages à distribuer (avec le même logiciel d'interrogation) leurs données rétrospectives ou historiques sur CD-ROMs, leurs clients ayant leurs services en ligne pour la recherche d'information très récente. Un serveur, H.W. Wilson, offre même la possibilité de passer directement de l'un (WilsonDisc) à l'autre (WilsonLine) pendant la même recherche.

Les prix des lecteurs CD-ROMs continuent à baisser. Déjà, les lecteurs CD-ROMs encastrés dans les micro-ordinateurs (comme pour les disquettes) sont apparus sur le marché. Le fait que des lecteurs hybrides ou multifonctionnels soient déjà disponibles créera aussi une force synergique dans l'ensemble des applications des technologies optiques. Celles-ci possèdent d'ailleurs une caractéristique unique en tant que support électronique d'information: elles ont plusieurs opportunités de marché et de nombreuses possibilités d'application. Si donc, pour une raison ou une autre, la croissance d'un sous-marché stagne, cela risque peu d'affecter les autres sous-marchés, créant ainsi un dynamisme continuel.

Le CD-ROM permet et encourage les activités de publication conjointes, soit des bases de données, des catalogues ou des fichiers totalement différents, soit des catalogues collectifs sur un même disque. Cela permet aussi aux producteurs de bases de données (incluant les bibliothèques) ne pouvant être en ligne, de s'associer pour les offrir sur des CD-ROMs. De plus, étant liées aux ordinateurs personnels, les technologies optiques offrent un marché beaucoup plus grand pour l'information électronique spécialisée, à ceux qui n'utiliseront jamais les services en ligne.

Pour plusieurs utilisateurs potentiels des disques optiques, les avantages réels ou perçus de ce nouveau support n'ont pas encore atteint le niveau qui les ferait passer de la recherche assistée par ordinateur (RAO) au disque optique. Le marché du disque compact est pourtant destiné à la consommation de masse grâce au lien du lecteur optique avec l'ordinateur personnel et le système d'opération. Les disques optiques sont de nouveaux outils à la portée de l'usager et lui offrent une plus grande souplesse d'interaction avec un grand volume d'informations; mais ils doivent d'abord être perçus comme des compléments aux autres modes d'accès à l'information.

Enfin, dans le cadre du défi Nord-Sud, considérant le besoin essentiel d'accès et d'échange d'information des pays en voie de développement, le CD-ROM permet de franchir une nouvelle étape dans les technologies utilisées pour la distribution d'information à grande échelle. Le CD-ROM ne nécessite pas de réseaux de télécommunications coûteux. II permet l'accès à de l'information électronique par la seule présence d'un microordinateur. Même les problèmes d'électricité peuvent être résolus par le recours à l'énergie solaire (piles) pour activer les micro-ordinateurs auxquels des lecteurs CD-ROMs à énergie solaire pourraient être connectés.

Ainsi, les technologies à la base du CD-ROM représentent des opportunités réelles et intéressantes pour les pays développés et les pays en développement car elles permettent à toute personne équipée d'un micro-ordinateur d'avoir accès à de grands volumes d'information à des coûts relativement faibles. La forte capacité de mémoire des CD-ROMs permet les publications conjointes ainsi que le partage des frais initiaux d'équipements et des coûts de distribution. En ce sens, les technologies optiques encouragent les activités de coopération et de collaboration dans la recension, l'échange et la diffusion d'information entre pays du nord et pays du sud, aussi bien dans les secteurs publics que privés.

Pour la bibliothèque, le disque optique représente une technologie polyvalente fort attrayante: catalogues, bases de données, ouvrages de référence, outils de marketing, outils de formation, etc. C'est une technologie encore 
jeune et au potentiel énorme, autant en termes de contenus qu'en termes d'accessibilité. Jusqu'à présent, la plupart des bibliothèques ont fait montre oh! surprise - d'un attentisme prudent ${ }^{26}$. Mais les initiatives et les expériences-pilotes commencent à se multiplier et continueront à se répandre au fur et à mesure que de nouveaux produits adaptés apparaîtront, que les normes seront répandues et acceptées par l'industrie, que les équipements s'amélioreront et que les coûts (surtout les coûts d'achat et d'abonnement aux produits) diminueront. Selon toutes les indications actuelles, ce n'est qu'une question de temps.

\section{ANNEXE 1}

\section{Sources d'information sur les technologies optiques et les CD-ROMs}

\section{Revues}

CD-ROM librarian

mensuel Meckler Publishing Corporation

11 Ferry Lane West

Westport, CT 06880

$65 \$$ US

\section{CD-ROM review}

1986- , mensuel

C.W. Communication Inc.

80 Elm Street

Peterborough, NH 03458

30 S US

Electronic and optical publishing review 1986- , trimestriel

Learned Information Ltd

143 Old Marlton Pike

Medford, NJ 08055

$68 \$$ US

\section{Laserdisk Professional}

1988- , bimensuel

Pemberton Press

11 Tannery Lana

Weston, CT 06883

$78 \$$ US

Optical information systems

1986- , bimensuel

Meckler Publishing Corporation

11 Ferry Lane West

Westport, CT 06880

95\$ US

\section{Comptes rendus de conférences}

\author{
INTERNATIONAL CONFERENCE ON \\ CD-ROM \\ 1986- , annuelle \\ Microsoft Corporation.
}

\section{OPTICAL PUBLISHING CONFERENCE \\ Annuelle.}

\section{Répertoires de bases de données}

\section{CD-ROM Sourcebook}

1986- , mises à jour mensuelles

Diversified Data Resources, Inc.

6609 Rosecroft Place

Falls Church, VA 22043

52 s US (2 volumes)

Guide to CD-ROMs in print

1987- , annuel (en juillet)

Meckler Publishing Corporation

11 Ferry Lane West

Westport, CT 06880

30 \$ US

Optical publishing directory

2 ième édition, 1987

Richard A. Bowers

Learned Information

143 Old Marlton Pike

Medford, NJ 08055

45 \$ US 
ANNEXE 2

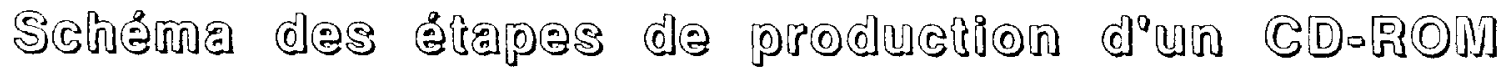

ÉTAPES

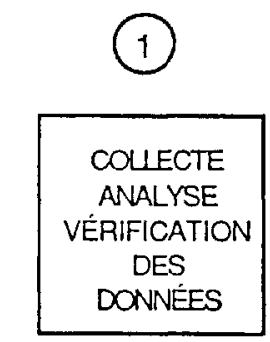

(2)

ORGANISATION

DES

DONNÉES
PROCESSUS

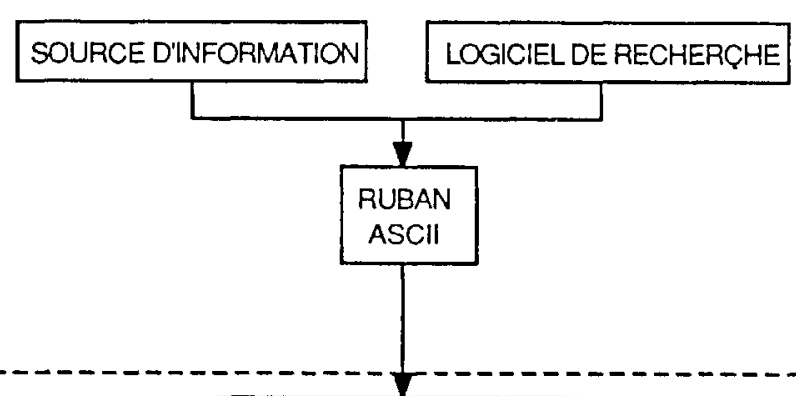

FICHIER RÉPERTOIRE

FICHIER MS-DOS

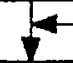

ORGANISATION SELON

LESNORMES

ANSI

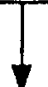

TEST DU LOGICIEL DINTERROGATION COMPATIBILTÉ/EFFICACITÉ

RUBAN POUR ENCODAGE DESDONNÉES
ACTEURS

FOURNISSEUR DE L'INFORMATION

FOURNISSEUR DU LOGICIEL

DINTERROGATION

ÉDITEUR DU DISQUE

FOURNISSEUR DE

L'INFORMATION 
S৫h ÉTAPES PROCESSUS

ACTEURS

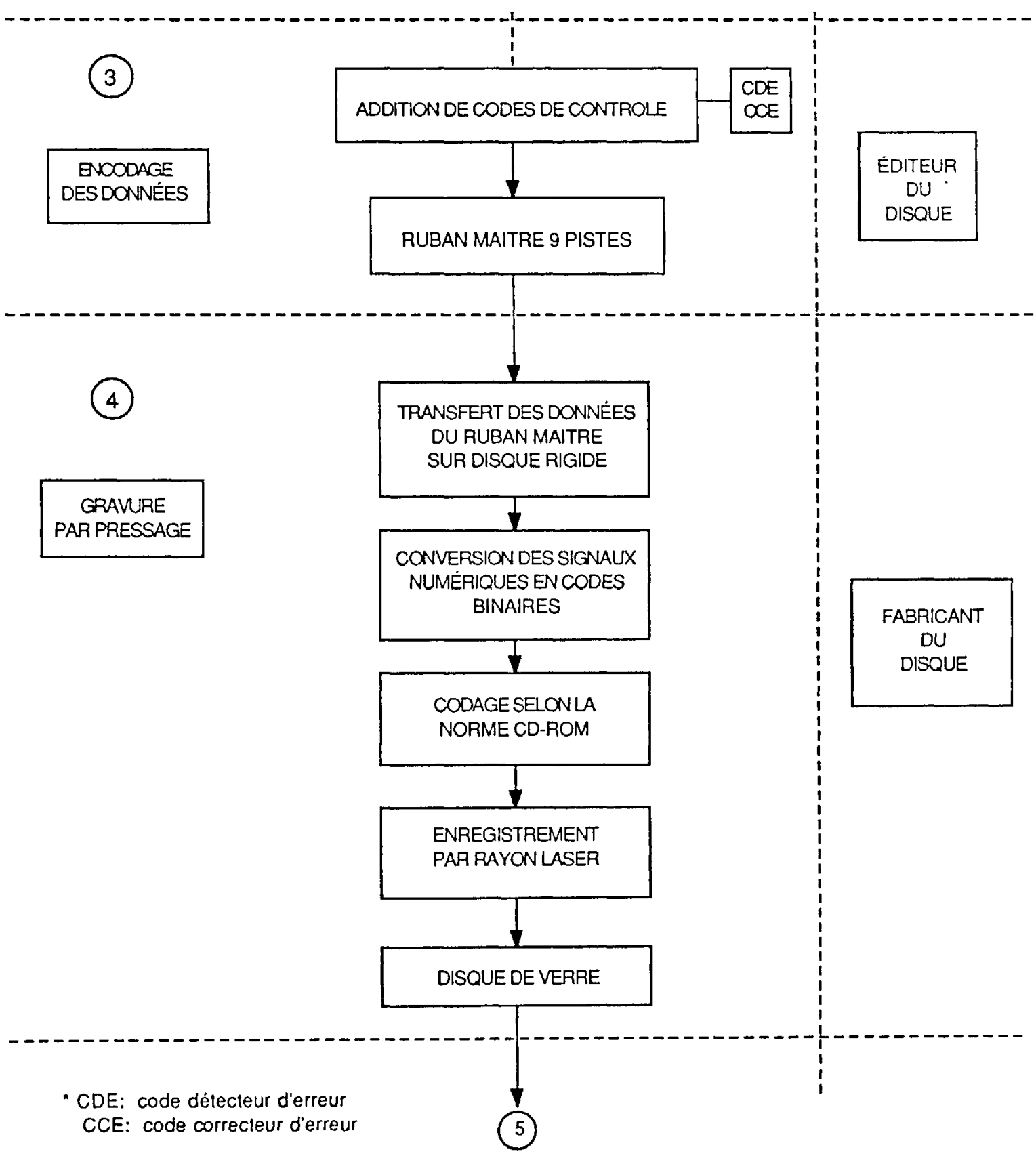




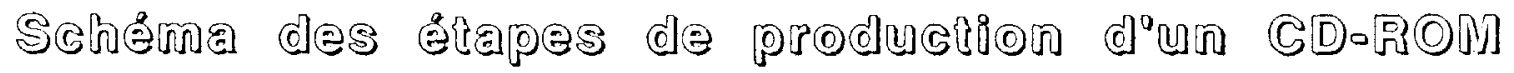
ÉTAPES PROCESSUS

ACTEURS

(5)

PRODUCTION DESDISQUES

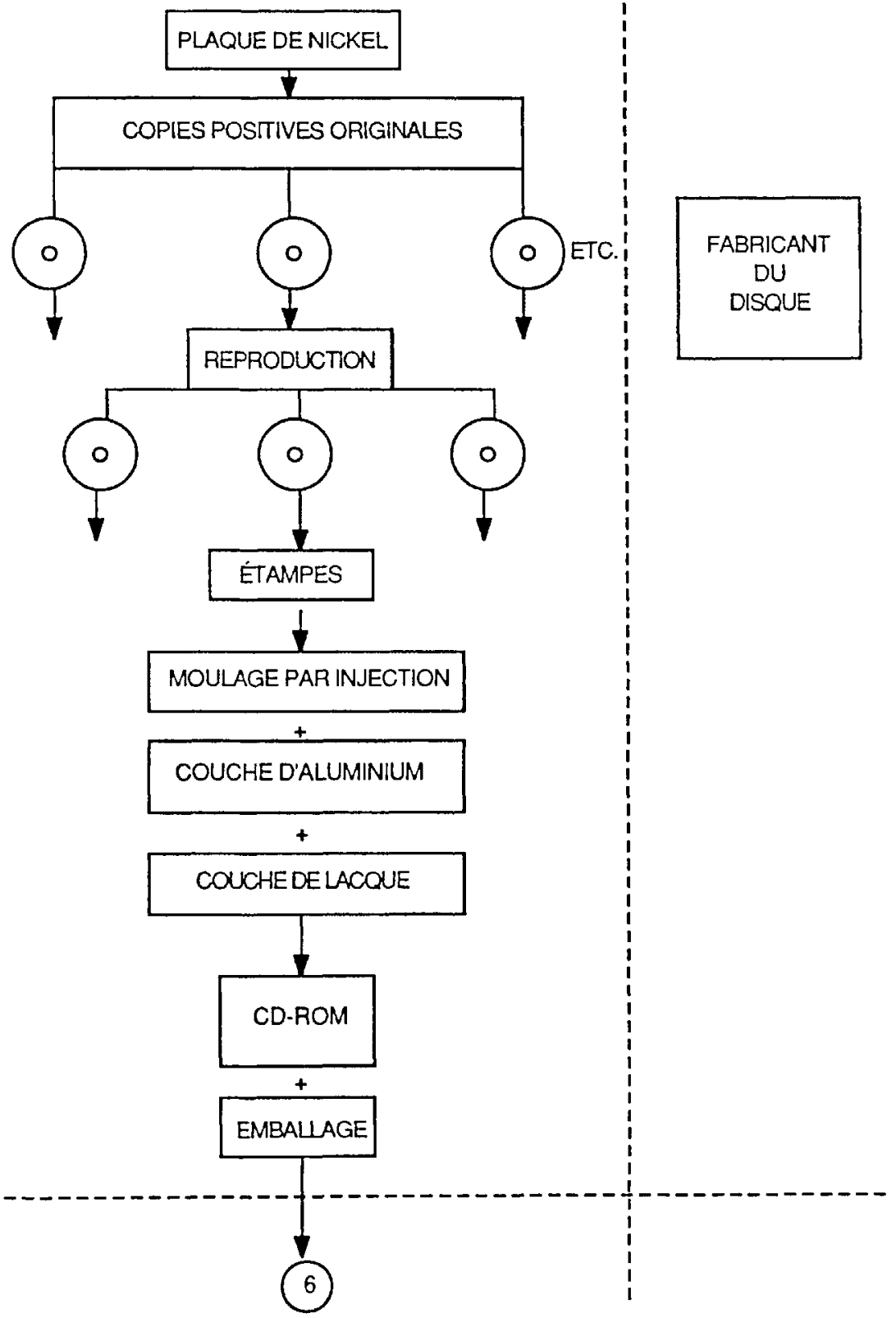




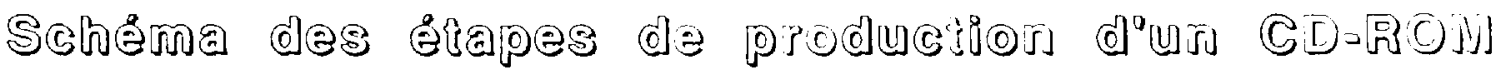

ÉTAPES

PROCESSUS

(6)

DISTRIBUTION

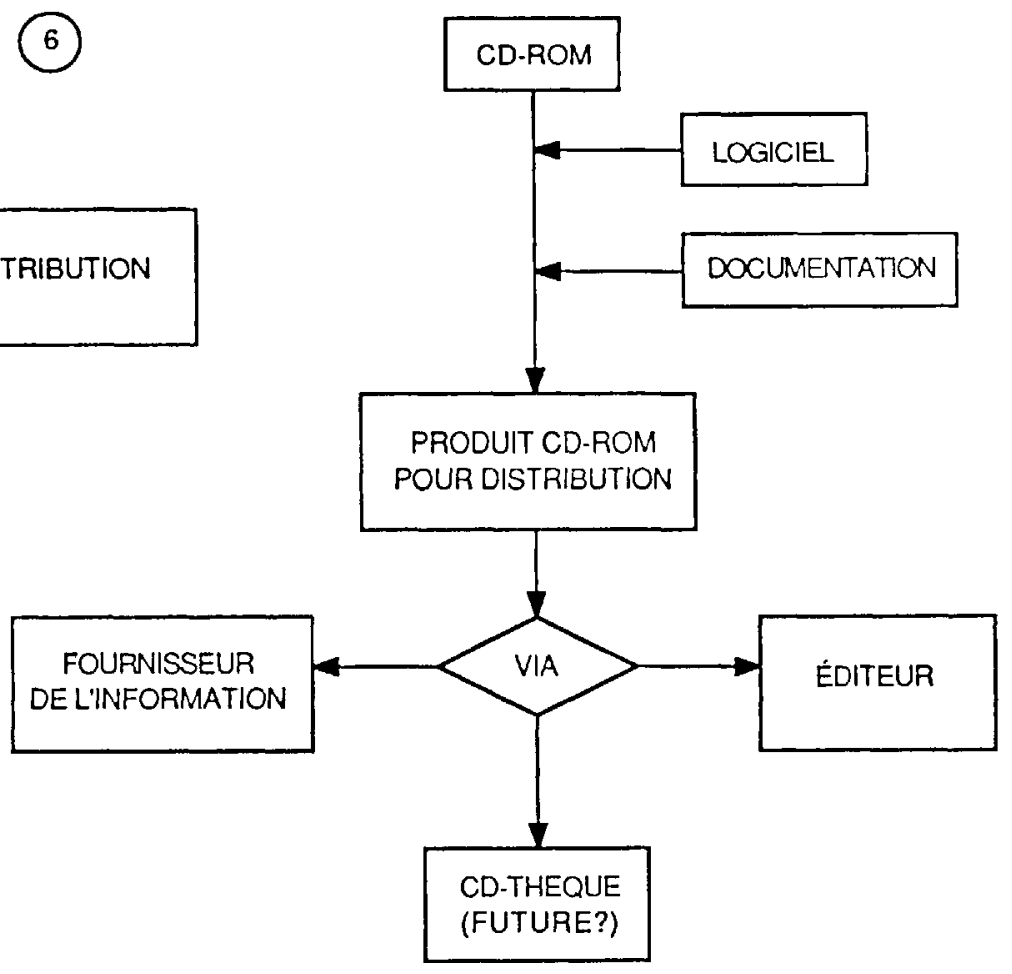

ACTEURS

FABRICANT

DU

DISQUE
ÉDITEUR

DU DISQUE

ov

FOURNISSEUR DE L'INFORMATION

o

CD-THEQUE (FUTURE?) 